\title{
Chapter 13 \\ Primate Infectious Disease Ecology: \\ Insights and Future Directions at the \\ Human-Macaque Interface
}

\author{
Krishna N. Balasubramaniam, Cédric Sueur, Michael A. Huffman, \\ and Andrew J. J. MacIntosh
}

\subsection{Introduction}

The expansion of human populations has increased interactions and conflict between humans and nonhuman primates (hereafter primates) throughout their range. Assessing the causal factors and thereby mitigating such conflict pose a major challenge for anthropologists, primatologists, and conservation biologists. This is because human-primate interactions are spatiotemporally variable in form and frequency (reviewed in Dickman 2012; Paterson and Wallis 2005). For instance, some of these interactions include (1) human-induced changes to primate habitat that lead to the fragmentation and decline of primate populations [e.g., Zanzibar red colobus monkeys (Procolobus kirkii): Siex 2005], (2) increased crop-raiding by primates leading to transactional costs on humans [e.g., Buton macaques (Macaca ochreata): Priston et al. 2012], (3) human-primate competition for space and resources [e.g., chimpanzees (Pan troglodytes): Hockings et al. 2012], (4) injuries to both humans and primates on account of direct aggression [e.g., rhesus macaques (Macaca mulatta): Southwick and Siddiqi 1994, 1998, 2011], and (5) primateinduced damage to human property and landscapes that generate transactional or opportunity costs to humans (Barua et al. 2013).

In comparison to such readily discernible negative effects, one outcome of conflict that is subtler and hence often goes undetected or unchecked is the

\footnotetext{
K. N. Balasubramaniam $(\bowtie)$

Department of Population Health and Reproduction, School of Veterinary Medicine, University of California at Davis, Davis, CA, USA

C. Sueur

IPHC, UMR 7178, Université de Strasbourg, CNRS, Strasbourg, France

e-mail: cedric.sueur@iphc.cnrs.fr
}

M. A. Huffman · A. J. J. MacIntosh

Primate Research Institute, Kyoto University, Kyoto, Japan

e-mail: huffman.michael.8n@kyoto-u.ac.jp 
acquisition and transmission of infectious diseases (Barua et al. 2013; Wolfe et al. 2007). Our shared evolutionary histories, along with physiological and behavioral similarities, make many primate species natural reservoirs of human parasites (Fiennes 1967; Nunn and Altizer 2006; Tutin 2000). Likewise, the acquisition of parasites from humans has led to disease outbreaks among free-living primates (Kaur and Singh 2009; Kaur et al. 2008, 2011; Nunn and Altizer 2006). From an ecological standpoint, free-living primates may acquire parasites from humans in many ways. For example, increasing epidemiological assessments continue to establish the sharing of parasites between humans and populations of socioecologically flexible primates like baboons and macaques which have become increasingly reliant on human-provisioned food or garbage in areas of overlap (Engel and Jones-Engel 2011; Engel et al. 2008; Jones-Engel et al. 2005). Humans may also indirectly influence primate exposure to parasites by altering the environment, which may potentially subdivide primate populations and change their behavioral and foraging strategies (Chapman et al. 2005, 2006a; Huffman and Chapman 2009). Third, wild primates may also sometimes be exposed to "spillovers" of parasites from international travelers during ecotourism and biological field research (Carne et al. 2017; Engel et al. 2008; Jones-Engel et al. 2005; Marechal et al. 2011; Muehlenbein and Ancrenaz 2010). Such a wide range of potential disease acquisition and transmission routes make human-primate interfaces hot spots for emerging infectious diseases (EIDs) (Nunn et al. 2008; Wolfe et al. 2007). This is especially significant in the light of the growing call for a global, transdisciplinary strategy to deal with zoonoses in both humans and animals (the One Health, hereafter $\mathrm{OH}$, concept: Destoumieux-Garzon et al. 2018; Zinsstag et al. 2011, 2015). Finally, human activities like agricultural and urban land development, tourism, and provisioning, aside from directly influencing exposure as stated above, may also influence variation in the susceptibility of primates to parasites once exposed, for example, by altering levels of stress and immune function (Chapman et al. 2006b; Marechal et al. 2011, 2016; Muehlenbein and Ancrenaz 2010).

In this chapter, we focus on how human-macaque interfaces, being hot spots for the transmission of a diverse array of parasites, present opportunities for humanprimate infectious disease ecology research. We first briefly outline the significance and primary objectives behind research on primate infectious disease ecology, highlighting the greater focus to date on research implementing such approaches to study wild primates in comparison to research at human-primate interfaces. We next reveal how macaques, and more broadly the variable nature of human-macaque interfaces, present opportunities to study human-primate disease transmission from a socioecological perspective (Engel and Jones-Engel 2011; Jones-Engel et al. 2005; Nahallage and Huffman 2013). We then provide a detailed account of previous studies we extracted from the online Global Mammal Parasite Database (Nunn and Altizer 2005; Stephens et al. 2017) that have detected parasites at human-macaque interfaces. Finally, we demonstrate how the implementation of novel conceptual frameworks like the Coupled Natural and Human Systems (An and Lopez-Carr 2012; Destoumieux-Garzon et al. 2018; Liu et al. 2007) and One Health concepts (Destoumieux-Garzon et al. 2018; Zinsstag et al. 2011, 2015), as well as the 
implementation of cutting-edge methodological approaches like Social Network Analyses (e.g., Drewe and Perkins 2015; Pasquaretta et al. 2014; Rushmore et al. 2017; VanderWaal and Ezenwa 2016) and community-level bipartite or multimodal Networks (e.g., Dormann et al. 2017; Gomez et al. 2013; Latapy et al. 2008), can address some of the critical gaps in these studies to offer key future directions for epidemiological research at these interfaces.

\subsection{Primate Infectious Disease Ecology}

An infectious disease is a disorder that is caused by an infectious agent, or in ecological terms a "parasite," that causes pathology in its host (MacIntosh 2016). In the ecological realm, a "parasite" is considered any organism that lives within (or on) another "host" organism, at some cost to the latter (MacIntosh 2016). For the remainder of the chapter, we deal with enteric parasites or "endoparasites" (hereafter just "parasites"), which live within the body of the host organism. These typically fall under seven major types of organisms. Five of these, specifically bacteria, viruses, rickettsia, prions, and fungi, are conventionally pathogenic microorganisms. The last two, protozoa and helminths, include both pathogenic and non-pathogenic species. All parasites typically disrupt the normal, homeostatic functioning of the body, both directly as a result of their own activity and indirectly by stimulating the host's immune system to produce a defensive response. They may do so by their sheer presence, by competing with host cells and symbiotic microbes, and, in extreme cases, by releasing toxins that increase the severity of diseases. Depending on their ecologies or life histories, parasites may enter hosts via their exposure to contaminated environmental sources such as food, water, and soil (e.g., enteric bacterial pathogens: Kilonzo et al. 2011; Sinton et al. 2007). They may also spread rapidly through host populations via mechanisms such as (1) direct host-to-host contact (e.g., respiratory viruses), (2) the sharing of common, contaminated environmental space or resources (e.g., enteric bacteria such as Salmonella sp., Shigella sp.), (3) exchange of body fluids (e.g. blood-borne pathogens like HIV and HPV), or via (4) vector-borne transmission [e.g., mosquitoes spreading malarial parasites (Plasmodium sp.)] (summarized in Engel and Jones-Engel 2011; Nunn and Altizer 2006).

Infectious disease ecology is a subfield that deals with the evolutionary and environmental factors that influence the exposure, acquisition, and transmission dynamics of parasites within and (more recently) between human and animal populations (Grenfell and Dobson 1995; Hudson et al. 2002). As we have now entered the Anthropocene epoch, human influence on the environment has generated an increased awareness of the importance of both public health and the conservation of natural ecosystems. So it is not surprising that over the last two decades in particular we have seen an incredible surge in research related to infectious disease ecology and evolution (reviewed in Huffman and Chapman 2009; Kappeler et al. 2015; MacIntosh and Frias 2016; Nunn 2012; Nunn and Altizer 2006), with 
interdisciplinary approaches drawing on theory and methods from several biological sciences including anthropology, evolutionary genetics, behavioral ecology, epidemiology, network theory, and statistics.

Nonhuman primates have served as especially useful model host systems in these endeavors (summarized in Huffman and Chapman 2009; Nunn 2012; Nunn and Altizer 2006). In addition to sharing evolutionary histories and, increasingly, ecological space with humans, primates also exhibit diverse forms of social systems, characterized by heterogeneity in group composition and size, dispersal patterns, foraging strategies, mating systems, and social structures (Hinde 1976; Kappeler and Van Schaik 2002; Sterck 1998; Thierry 2007a). For these reasons, they are physiological, ecological, and behavioral model host systems for infectious disease research (MacIntosh 2016). There is now consensus among scientists that the evolutionary, ecological, and social diversity of free-living primates is impacted by (or indeed impact) the risk of acquisition and transmission of parasites (Sueur et al. 2018).

Broadly, empirical research on primate infectious disease ecology to date has had five major foci. First, in studies related to (1) parasite-host co-evolution, evolutionary anthropologists have attempted to establish links between the phylogenetic relationships of parasites and their primate hosts (MacIntosh and Frias 2016; Nunn 2011; Nunn and Altizer 2006; Petrášová et al. 2011; Vallo et al. 2012). Second, studies on (2) primate parasite socioecology, in addition to the relative role(s) of resource abundance, predation pressure, and infanticidal risk, have also begun to examine the role of parasites in shaping the evolution of primate group sizes and social network structure (Chapman et al. 2009; Nunn et al. 2011; meta-analyses by Griffin and Nunn 2012; Nunn et al. 2015; Patterson and Ruckstuhl 2013; Rifkin et al. 2012). Conversely, the idea that group-living and social structure may also impact the diversity and prevalence of parasites in hosts (Drewe and Perkins 2015; VanderWaal and Ezenwa 2016) has led to such socioecological approaches to also focus on the identification of potential "super spreaders" or "social bottlenecks" of infection (Balasubramaniam et al. 2016, 2018; Duboscq et al. 2016; Griffin and Nunn 2012; MacIntosh et al. 2012; Romano et al. 2016). Other studies have used agent-based models to predict the prevalence and transmission of parasites through artificial primate groups and networks (Griffin and Nunn 2012; Nunn et al. 2015). Yet social life does not always equate to disease transmission or threats to homeostasis. Indeed, studies on both captive and wild primates that assess the links between (3) infection risk and sociality, stress, and immune function have tested the opposite paradigm, i.e., that possessing strong, diverse social connections, rather than increasing the risk of pathogenic acquisition, may function to socially buffer some primates against infection (Balasubramaniam et al. 2016; Duboscq et al. 2016; Sapolsky et al. 2000; Young et al. 2014). More research has focused on the impact of (4) parasites in primate conservation and management-while some deal with the implications of introduced species on the prevalence and diversity of parasites in indigenous primates (Petrášová et al. 2010, 2011), other research has attempted to quantify differences in parasite richness or diversity in primates living in varying degrees of human influence or in relation to their threatened status(es) (Bublitz et al. 
2015; Chapman et al. 2006a; Gillespie et al. 2005; Goldberg et al. 2007; Kowalewski et al. 2011). Finally, emerging lines of research have focused on (5) primate counterstrategies, including avoidance behaviors to minimize exposure to parasites (Amoroso et al. 2017; Poirotte et al. 2017, 2019; Sarabian and MacIntosh 2015; Sarabian et al. 2017), and self-medication that removes or minimizes the impact of an infection on the host (Huffman 2016).

To date, much of the empirical work related to primate infectious disease ecology has focused on wild or red-listed primate populations (reviewed in Frias and MacIntosh 2018). Aside from habitat loss and fragmentation (Hussain et al. 2013), red-listed populations also face the risk of extinction on account of infectious diseases transmitted from humans or livestock (reviewed in Frias and MacIntosh 2018). In comparison, less research has focused on the relationship between host socioecology and transmission of parasites between humans and free-living primates at overlapping interfaces (Kaur and Singh 2009). This is despite the wide recognition that humans and primates strongly influence each other's biology, behavior, and health (Fuentes 2012; Fuentes and Hockings 2010) and that such human-primate interfaces are also potential sources of EIDs (Jones-Engel et al. 2005; Nunn et al. 2008; Wolfe et al. 2007).

\subsection{Human-Macaque Interfaces}

The genus Macaca is the most diverse, geographically widespread, and ecologically successful group of primates (Cords 2013; Thierry 2007a, b). They constitute 23 extant species, which range from North Africa in the West (Barbary macaques: M. sylvanus), across the Indian subcontinent [e.g., rhesus macaques (M. mulatta), bonnet macaques (M. radiata)], China [e.g., rhesus macaques (M. mulatta), Tibetan macaques (M. thibetana)], and Southeast Asia [e.g., long-tailed macaques (M. fascicularis), Sulawesi macaque species (e.g., M. nigra, M. tonkeana)], and up to Japan in the Far East [Japanese macaques (M. fuscata)] (Thierry 2007a, b). Across this range, their ecological flexibility is evidenced by the fact that macaque species, and indeed populations of the same species, inhabit a wide variety of habitats, from tropical rainforests to snowcapped mountains and from dry scrub forests to urbanized human settlements (Cords 2013; Gumert et al. 2011; Thierry 2007a, b).

In nature, all macaque species show broadly similar social organization [but see Sinha et al. (2005) for an exception] — they live in multi-male multi-female social groups in which females are philopatric and males disperse from their natal groups (Cords 2013; Thierry 2013). At the same time, they show a remarkable degree of inter- and intraspecific variation in the structure of social relationships, ranging from despotic, nepotistic societies with steep dominance hierarchies and modular, centralized, and kin-biased social networks (e.g., rhesus macaques, Japanese macaques) to tolerant or egalitarian societies characterized by shallower dominance relationships and dense, decentralized, and well-connected social networks (e.g., Sulawesi 


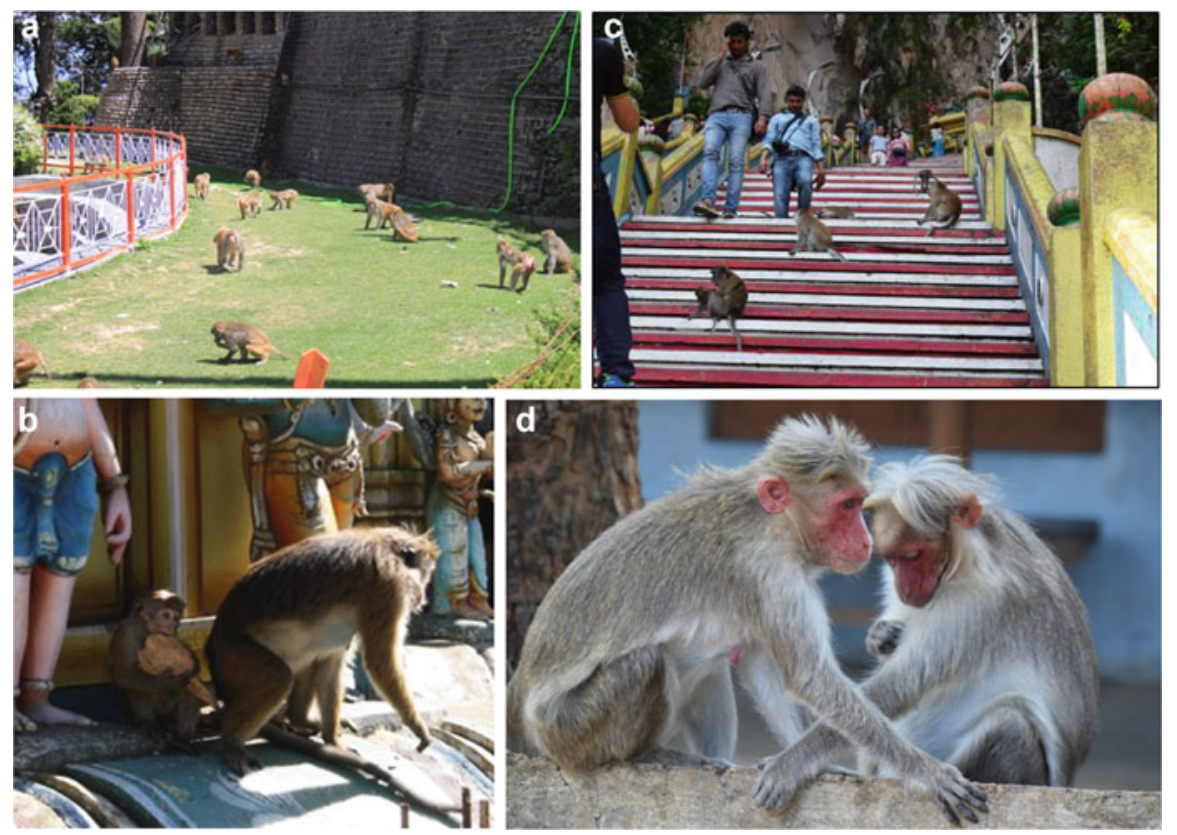

Fig. 13.1 Macaques at human-macaque interfaces, specifically (a) rhesus macaques in Himachal Pradesh, Northern India; (b) long-tailed macaques in Kuala Lumpur, Malaysia; (c) toque macaques in Colombo, Sri Lanka; (d) bonnet macaques in Kerala, Southern India. Photo credits: (a), (b) and (d): K. N. Balasubramaniam; (c): M. A. Huffman

crested macaques: Balasubramaniam et al. 2012; Thierry et al. 2008; Sueur et al. 2011a).

More pertinently, macaques also vary in the extent to which they show adaptive or maladaptive responses to human disturbance and anthropogenic landscapes, i.e., along a spectrum of overlap at human-macaque interfaces (Priston and McLennan 2013; Radhakrishna and Sinha 2011; Radhakrishna et al. 2013). At the upper end of this spectrum lie rhesus and long-tailed macaques (Fig. 13.1a, b). Large populations of these "weed" species, categorized as "Least Concern" by IUCN since 2010 (IUCN 2019), gravitate toward and even preferentially exploit human settlements (Jaman and Huffman 2013; Southwick and Siddiqi 1994, 2011; Southwick et al. 1983). Long-tailed macaques are even listed among the IUCN Invasive Species Specialist Group's (ISSG) top 100 invasive species in the world (Lowe et al. 2000). Thus, they inhabit a variety of human-macaque interfaces: from buffer zones of ecotourism in national parks, to agricultural fields bordering rural villages, to urbanized cities like Delhi, Dhaka, and Kuala Lumpur (Fig. 13.1a, b). Other species like bonnet macaques and toque macaques ( $M$. sinica) are not far behind, with both wild and semi-urban populations that inhabit the smaller town-, temple-, and university campus-interfaces of Southern India and Sri Lanka, respectively (Huffman et al. 2013a; Nahallage and Huffman 2013; Nahallage et al. 2008; Radhakrishna 
et al. 2013; Ram et al. 2003; Sinha et al. 2005) (Fig. 13.1c, d). Yet some of these species, like toque macaques, remain listed as "vulnerable" or "endangered" on account of the negative effects of ecotourism and habitat loss throughout their range (IUCN 2019). Finally, some less ecologically flexible species like lion-tailed macaques (M. silenus), Tibetan macaques (M. thibetana), and Sulawesi crested macaques (M. nigra) are still exposed to the negative impact of human activity in the form of habitat loss affecting their socioecology (Kumara et al. 2014; Singh et al. 2001), ecotourism-related stressors and mortality rates (Berman et al. 2007; Marechal et al. 2011), and hunting for bush-meat impacting mortality rates (Kyes et al. 2012; Palacios et al. 2012; Riley 2007; Riley and Fuentes 2011). Indeed, many of these species are classified as being "endangered" or "critically endangered" as a result (IUCN 2019).

The rise of ethnoprimatology as a subfield of biological anthropology has occurred simultaneously with the rise of primate infectious disease ecology. Specifically, ethnoprimatology is related to understanding how humans and primates impact each other's niche construction, behavioral biology, and health-related outcomes (Dore et al. 2017; Fuentes 2012; Fuentes and Hockings 2010). Unsurprisingly, human-macaque interfaces in North Africa, India, Sri Lanka, and Southeast Asia have been the primary foci of most ethnoprimatological research, with some more recent studies in Africa also having been conducted on baboons (Fehlmann et al. 2016; Kaplan et al. 2011; Hoffman and O'Riain 2012), chimpanzees (Hockings et al. 2012), and lemurs (Loudon et al. 2017). To date, this work has revealed that the nature, frequency, and severity of interactions and conflict at human-macaque interfaces vary broadly by context (Radhakrishna and Sinha 2011; Radhakrishna et al. 2013). For instance, across the Indian subcontinent, China, and Southeast Asia, conflict is heavily influenced by whether macaques also play more positive roles with resident and/or visiting human communities, e.g., monkeys as religious symbols, pets, trade commodities, or tourist attractions (Jones-Engel et al. 2004; Nahallage and Huffman 2013; Radhakrishna et al. 2013). At the same time, some intrinsic characteristics of macaques, including the age-sex class, personalities of individuals, and/or species-typical adaptive responses, have also been shown to influence interface interactions (Beisner et al. 2014; Fuentes 2006; Marechal et al. 2011; Sha et al. 2009). Such variation in human- and macaque-specific features across interfaces generates a broad variety of direct and indirect interactions, such as (1) human provisioning of macaques, (2) macaques using anthropogenic landscape features (e.g., buildings, fences, water tanks), (3) mutual contact- and non-contact aggression, (4) the exchange of body fluids like blood and saliva, (5) the fragmentation of macaque populations on account of the loss of natural habitat, (6) the hunting and consumption of macaques by humans as bush-meat, and (7) the use of macaques as pets, trade commodities, or tourist attractions (Fuentes et al. 2011; Gumert et al. 2011; Hussain et al. 2013; Jones-Engel et al. 2004; Radhakrishna et al. 2013; Riley and Fuentes 2011; Riley 2003). Naturally, the dynamic nature of such environments provides myriad mechanisms for the acquisition and transmission of parasites (Engel and Jones-Engel 2011; Nunn 2012). 


\subsection{Parasites at Human-Macaque Interfaces}

To extract and review previous studies that report parasites among free-living macaque populations at human-macaque interfaces, we relied on the Global Mammal Parasite Database (or GMPD, Version 2.0: Stephens et al. 2017). The GMPD is a compilation of studies that report disease-causing organisms-bacteria, viruses, protozoa, helminths, and fungi-isolated from wild or free-living populations of some of the major mammalian taxa, specifically ungulates, carnivores, and primates (Nunn and Altizer 2005; Stephens et al. 2017). The database now contains 24,000 records, from over 2700 literature sources including journal articles, books and book chapters, and reports at conference proceedings. Records may be filtered on the basis of different parasite or host-specific characteristics, such as taxonomic categories, geographic location, and mode of transmission.

A search of the GMPD database filtered by host genus (macaques) and type of parasite (bacteria, viruses, helminths, and protozoa) revealed 570 records from across 80 different studies. Figure 13.2 indicates the distribution of these records by study period. Aside from the general geographic location, the GMPD does not offer more specific filtering options that aid in the classification of studies in accordance with socioecological conditions under which they were conducted. So, we manually screened for "human-macaque interface" studies as those among the above studies that reported one or more of the following: (1) the direct transmission of these agents between humans and macaques in either direction (e.g., contact

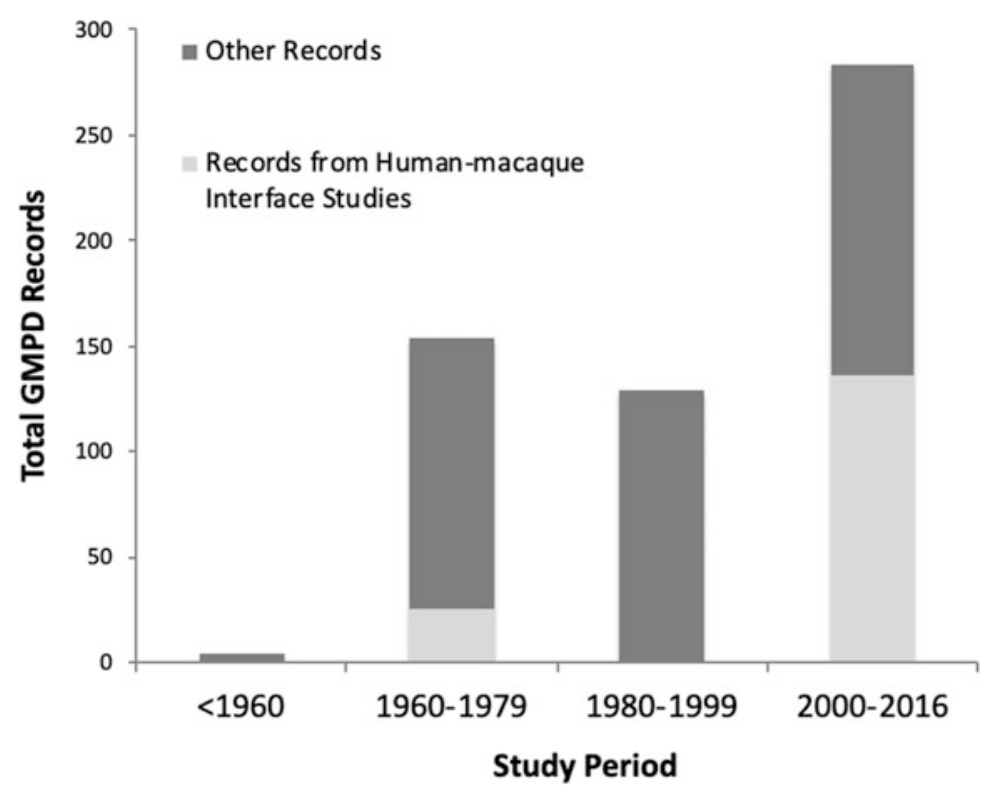

Fig. 13.2 Records of parasites reported among free-living macaque populations from studies in the Global Mammal Parasite Database (GMPD) 
aggression or provisioning, pet macaques released into free-living populations), (2) the possible or potential transmission of such agents, or (3) the indirect impact of humans or anthropogenic factors (e.g., habitat fragmentation, livestock, macaque foraging on provisioned food) being identified to have influenced the acquisition of these parasites among macaques. Since this chapter primarily deals with the socioecological impact of human-macaque interfaces on disease risk, we also did not include studies on the phylogenetic co-evolutionary roots of parasites and their primate hosts.

These criteria led to the extraction of 161 (out of 570) macaque records, the vast majority of which were dated post 2000 (130 out of 161: Fig. 13.2). These records were spread across nine cited studies conducted on six different species of macaques. In Table 13.1, we summarize information from these studies, providing details on the parasites isolated, host macaque species, geographic location, prevalence and the number of individuals sampled (where the information is available), and the type of acquisition reported or speculated. Unsurprisingly, viral agents dominate this list with 88 of the 161 entries (or 55\%) from 4 citations. During the last two decades, several zoonotic viruses have been described and studied in nonhuman primates in Africa and Asia from evolutionary and virulence perspectives (e.g., Ebola in great apes, reviewed in Leendertz et al. 2017; respiratory viruses in macaques, reviewed below). From a socioecological standpoint, studies on macaques have revealed strong associations between the frequency of intense human-macaque contact behaviors that involve the exchange of body fluids (e.g., aggressive bites and scratches) and the prevalence of respiratory viruses. Early work showed that wildcaught rhesus macaques in Northern India that had the highest degrees of exposure to human contact were also the most likely to show blood serum antibodies against human respiratory viruses (Shah and Southwick 1965). Later work in Nepal revealed correlations between the frequency of intense contact interactions with humans like aggressive bites and scratches and the seroprevalence of respiratory viruses such as the simian foamy virus (SFV), simian type-D virus, Cercopithecine herpesvirus-1 (CHV-1), and simian virus-40 (SV-40) (Jones-Engel et al. 2006) among rhesus macaques living in human settlements. In comparison, the Barbary macaques of Gibraltar, which engage in less intense aggression and have lower rates of contact bites in comparison to the Asian rhesus populations, showed a markedly lower seroprevalence (or were even seronegative) of these same respiratory viruses (Engel et al. 2008). More recently, humans traveling with performing "pet" rhesus macaques were found to indirectly influence the genetic structure and translocation of macaque SFV across rhesus populations in Bangladesh (Feeroz et al. 2013). Finally, other work not included in the GMPD has recorded the prevalence of macaque-borne viral pathogens like SFV and retroviruses among humans that regularly come into contact with these populations (Jones-Engel et al. 2005).

Gastrointestinal protozoa (36 entries out of 161, or 22\%) and helminths (26 entries out of 161 , or $16 \%$ ) were the next most commonly reported parasites among the human-macaque interface studies examined. In nature, these are among the most commonly occurring parasites in wild primates (Huffman and Chapman 2009; Nunn and Altizer 2006). Yet, a few ecological assessments of human-perturbed landscapes 


\begin{tabular}{|c|c|c|c|c|c|c|c|c|c|c|c|c|c|}
\hline 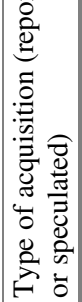 & 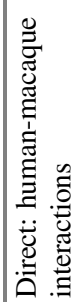 & 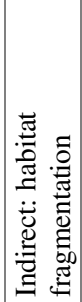 & 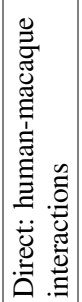 & 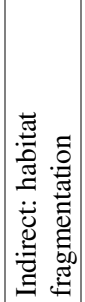 & 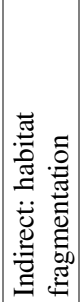 & 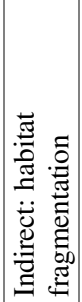 & 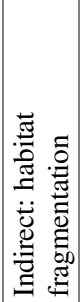 & 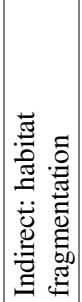 & 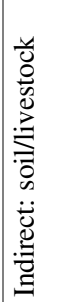 & 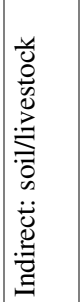 & 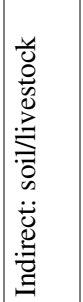 & 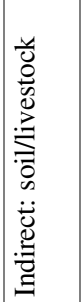 & 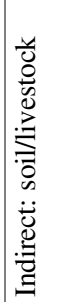 \\
\hline $\overrightarrow{0}$ & ๙ิ? & ָ̃ & $\stackrel{1}{\circ}$ & ڤ̊ & 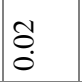 & $\stackrel{\sim}{\tilde{2}}$ & $\stackrel{\sim}{\tilde{c}}$ & $\stackrel{0}{8}$ & $\tilde{n}$ & 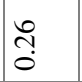 & $\stackrel{2}{0}$ & ڤે̀ & în. \\
\hline $\begin{array}{l}\dot{00} \\
\overline{0} \\
\end{array}$ & $\infty$ & $\infty$ & $\bar{\infty}$ & کั. & ô. & ợ & ợ & ố & $\bar{\infty}$ & $\bar{\infty}$ & $\bar{\infty}$ & $\infty$ & $\bar{\infty}$ \\
\hline 両 & $r$ & $r$ & $r$ & $\stackrel{+}{\circ}$ & $\stackrel{+}{0}$ & $\stackrel{+}{\ominus}$ & $\stackrel{+}{\stackrel{0}{0}}$ & $\stackrel{+}{\ominus}$ & $\frac{9}{r}$ & $\stackrel{9}{r}$ & $\stackrel{9}{r}$ & $\stackrel{9}{r}$ & $\stackrel{9}{r}$ \\
\hline $\begin{array}{l}\tilde{0} \\
\stackrel{\Xi}{0} \\
0 \\
0\end{array}$ & 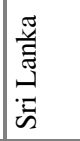 & 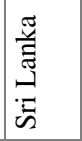 & 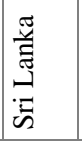 & 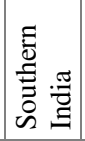 & 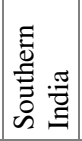 & 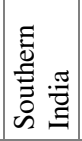 & 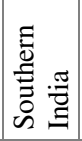 & 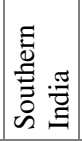 & 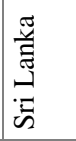 & 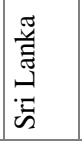 & 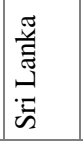 & 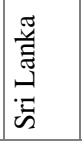 & 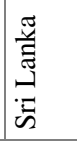 \\
\hline 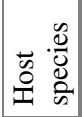 & 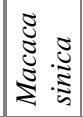 & 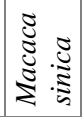 & 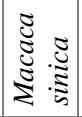 & 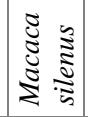 & 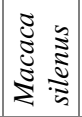 & 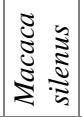 & 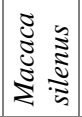 & 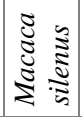 & 芯 & 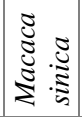 & 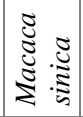 & 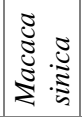 & 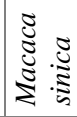 \\
\hline 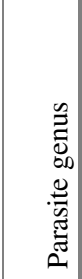 & 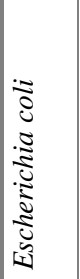 & 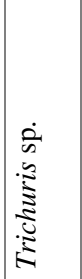 & 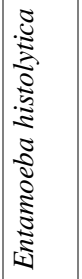 & 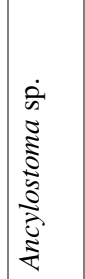 & 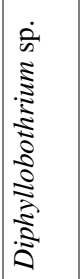 & 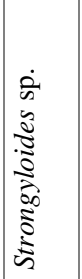 & 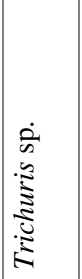 & 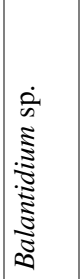 & 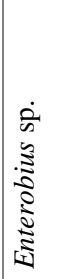 & 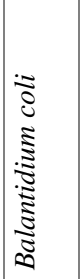 & 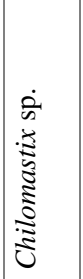 & 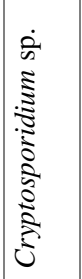 & 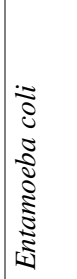 \\
\hline 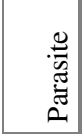 & 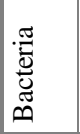 & 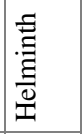 & $\begin{array}{l}\widetilde{0} \\
0 \\
0 \\
0 \\
0\end{array}$ & 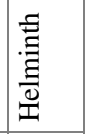 & 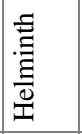 & 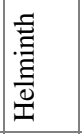 & 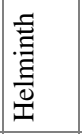 & $\begin{array}{l}\tilde{N} \\
0 \\
0 \\
0 \\
0\end{array}$ & 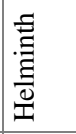 & 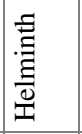 & 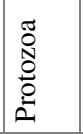 & $\begin{array}{l}\tilde{N} \\
0 \\
0 \\
0 \\
2\end{array}$ & 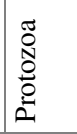 \\
\hline 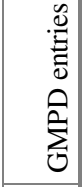 & হ & হু & হু & $\underset{\substack{\infty \\
\infty}}{\stackrel{2}{\infty}}$ & $\begin{array}{l}\infty \\
\infty \\
\infty\end{array}$ & $\frac{n}{\infty}$ & 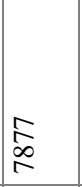 & $\begin{array}{l}\infty \\
\infty \\
\infty\end{array}$ & 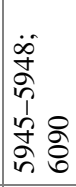 & 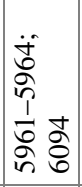 & 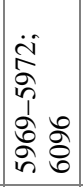 & $\begin{array}{l}\text { वे } \\
\text { in } \\
\frac{1}{n} \\
\text { nn }\end{array}$ & 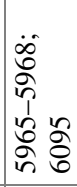 \\
\hline 总 & 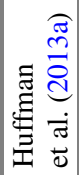 & 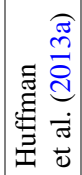 & 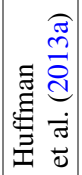 & 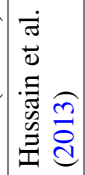 & 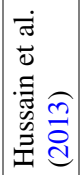 & 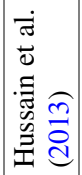 & 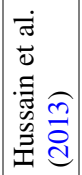 & 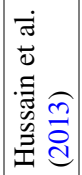 & 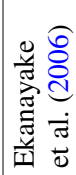 & 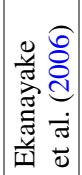 & 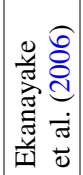 & 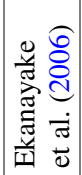 & 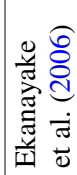 \\
\hline
\end{tabular}


13 Primate Infectious Disease Ecology: Insights and Future Directions at...

\begin{tabular}{|c|c|c|c|c|c|c|c|c|c|c|c|}
\hline 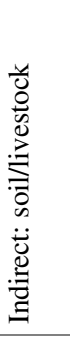 & 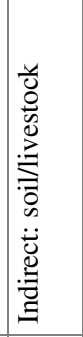 & 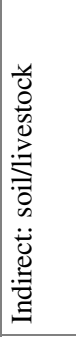 & 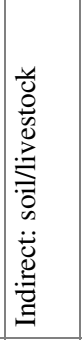 & 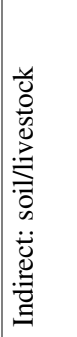 & 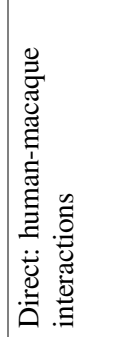 & 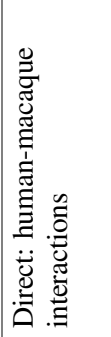 & 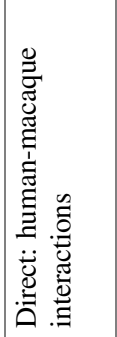 & 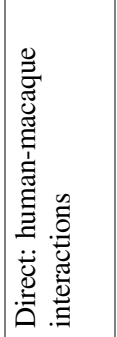 & 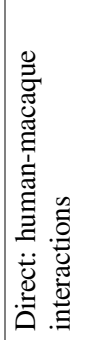 & 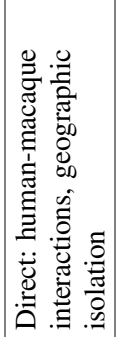 & 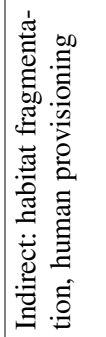 \\
\hline స̂. & @̊ & î & సิ & $\stackrel{8}{0}$ & $\stackrel{8}{8}$ & $\stackrel{8}{8}$ & 8 & 8 & กิ? & $\hat{\delta}$ & $\stackrel{n}{o}$ \\
\hline $\bar{\infty}$ & $\infty$ & $\bar{\infty}$ & $\infty$ & $\infty$ & $\begin{array}{l}0 \\
\dot{i} \\
1\end{array}$ & $\begin{array}{l}0 \\
i \\
i\end{array}$ & $\begin{array}{l}0 \\
\dot{1} \\
1\end{array}$ & $\begin{array}{l}0 \\
i \\
i\end{array}$ & $\begin{array}{l}0 \\
\dot{1} \\
1\end{array}$ & & $\begin{array}{l}m \\
\infty \\
\infty\end{array}$ \\
\hline$\stackrel{9}{r}$ & $\stackrel{9}{r}$ & $\frac{9}{2}$ & $\stackrel{9}{r}$ & $\stackrel{9}{r}$ & ల & ల) & $\stackrel{0}{\infty}$ & ల & ల & & $\stackrel{\hat{\imath}}{\hat{\imath}}$ \\
\hline 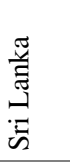 & 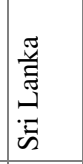 & 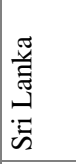 & 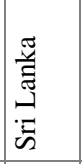 & 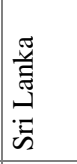 & 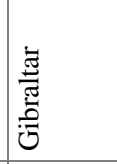 & 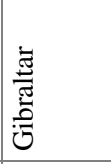 & 苞 & 吾 & 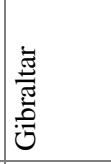 & 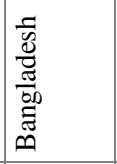 & $\begin{array}{l}\tilde{a} \\
\overline{\tilde{z}} \\
\end{array}$ \\
\hline 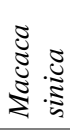 & 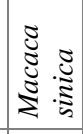 & 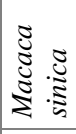 & 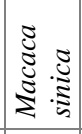 & 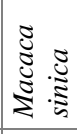 & 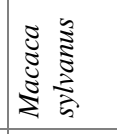 & 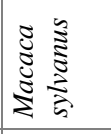 & 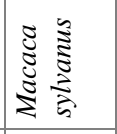 & 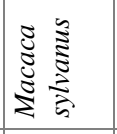 & 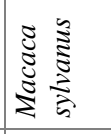 & 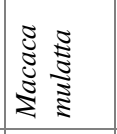 & 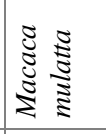 \\
\hline 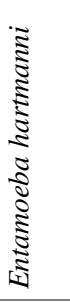 & 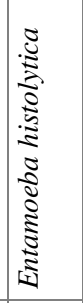 & 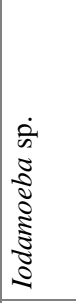 & 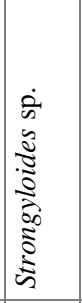 & 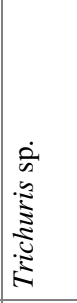 & 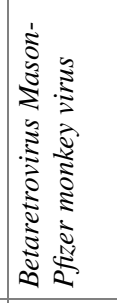 & 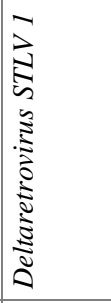 & 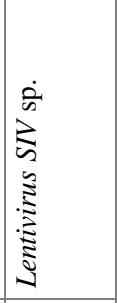 & 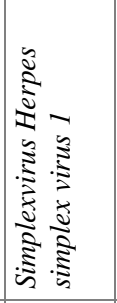 & 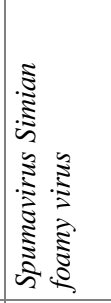 & 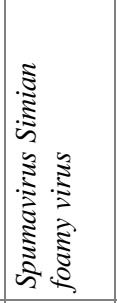 & 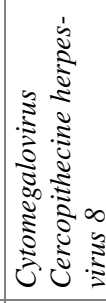 \\
\hline $\begin{array}{l}\pi \\
0 \\
0 \\
0 \\
0 \\
0\end{array}$ & $\begin{array}{l}\text { : } \\
0 \\
0 \\
0 \\
0 \\
0\end{array}$ & $\begin{array}{l}\approx \\
0 \\
0 \\
0 \\
0 \\
0\end{array}$ & 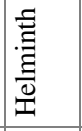 & 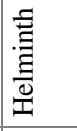 & $\stackrel{气}{\Xi}$ & $\stackrel{\mathscr{E}}{\supset}$ & $\stackrel{0}{.}$ & 蛋 & $\stackrel{0}{.}$ & $\stackrel{0}{.}$ & 兰 \\
\hline 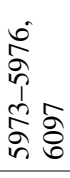 & $\begin{array}{l}\ddot{0} \\
\infty \\
i n \\
\hat{1} \\
\hat{2} \\
\hat{n} \\
0 \\
0\end{array}$ & 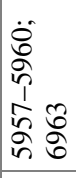 & 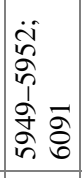 & 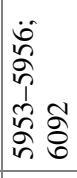 & 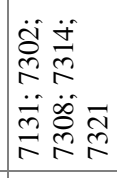 & 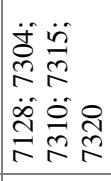 & 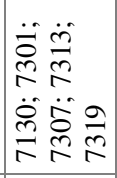 & 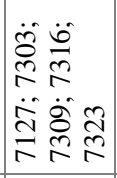 & 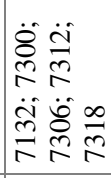 & 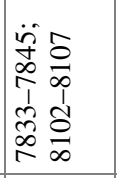 & 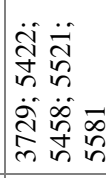 \\
\hline 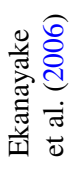 & 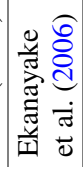 & 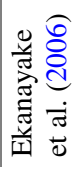 & 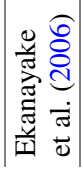 & 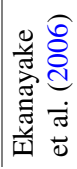 & 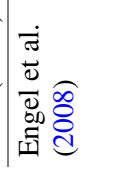 & 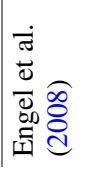 & 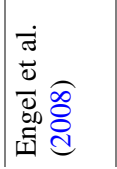 & 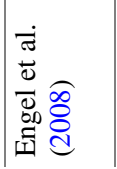 & 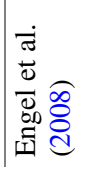 & 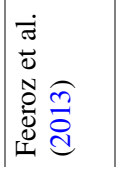 & 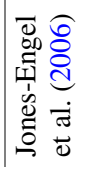 \\
\hline
\end{tabular}




\begin{tabular}{|c|c|c|c|c|c|c|c|c|c|c|c|}
\hline 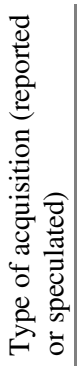 & 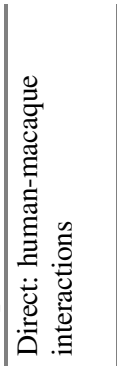 & 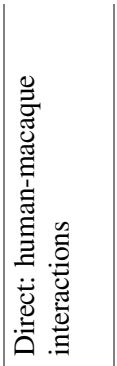 & 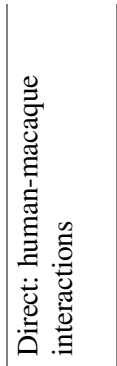 & 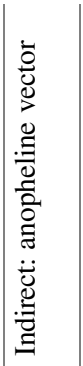 & 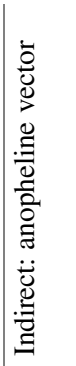 & 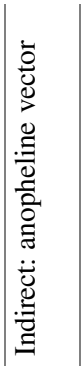 & 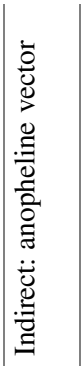 & 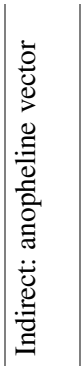 & 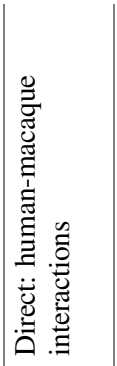 & 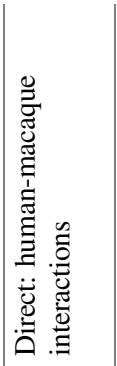 & 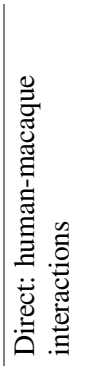 \\
\hline$\vec{D}$ & \& & $\begin{array}{l}\text { ț } \\
\stackrel{0}{0}\end{array}$ & $\hat{a}$ & $\stackrel{f}{f}$ & $\stackrel{\infty}{\stackrel{0}{0}}$ & $\underset{0}{0}$ & 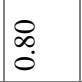 & $\begin{array}{l}2 \\
0 \\
0\end{array}$ & $\stackrel{8}{8}$ & $\stackrel{8}{8}$ & 8 \\
\hline 0.0 & $\begin{array}{l}m \\
\infty \\
\infty\end{array}$ & $\begin{array}{l}m \\
\infty \\
\infty\end{array}$ & $\begin{array}{l}m \\
\infty \\
\infty\end{array}$ & & & & & & $\infty$ & $\infty$ & $\infty$ \\
\hline$\dot{\tilde{J}}$ & 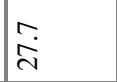 & $\stackrel{i}{i}$ & 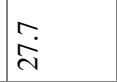 & & & & & & $\underset{\sim}{\infty}$ & $\stackrel{\infty}{\sim}$ & $\infty$ \\
\hline 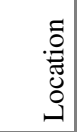 & 营 & 㤩 & 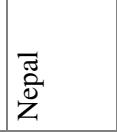 & $\begin{array}{l}8 \\
\stackrel{D}{E} \\
\stackrel{0}{0} \\
\infty\end{array}$ & 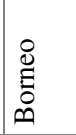 & 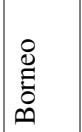 & $\begin{array}{l}\dot{D} \\
\stackrel{D}{E} \\
0 \\
\infty\end{array}$ & 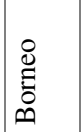 & 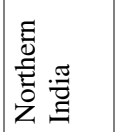 & 泀 & 胥 \\
\hline 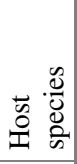 & 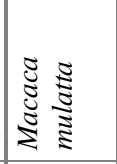 & 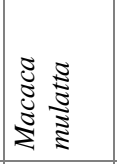 & 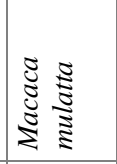 & 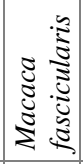 & 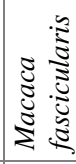 & 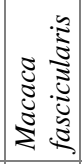 & 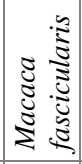 & 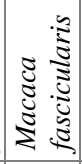 & 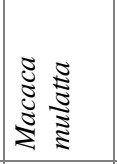 & 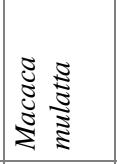 & 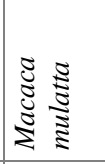 \\
\hline 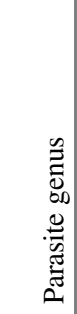 & 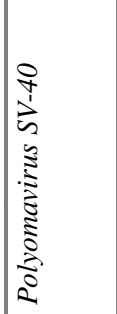 & 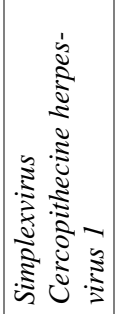 & 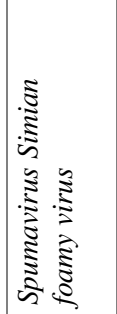 & 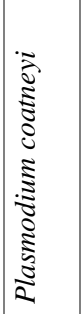 & 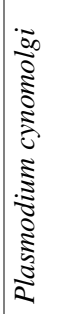 & 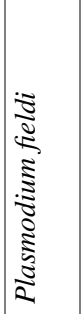 & 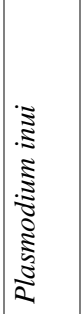 & 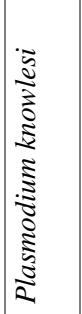 & 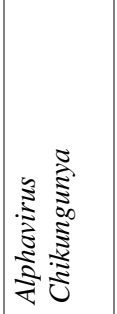 & 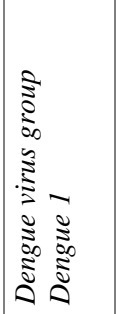 & 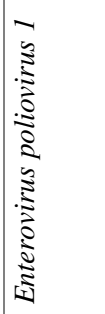 \\
\hline 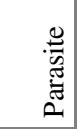 & $\stackrel{0}{\Xi}$ & $\stackrel{0}{\xi}$ & 茎 & $\begin{array}{l}\tilde{0} \\
0 \\
0 \\
0 \\
0 \\
0\end{array}$ & $\begin{array}{l}0 \\
0 \\
0 \\
0 \\
0 \\
01\end{array}$ & $\begin{array}{l}0 \\
0 \\
0 \\
0 \\
0 \\
0\end{array}$ & $\begin{array}{l}0 \\
0 \\
0 \\
0 \\
0 \\
0\end{array}$ & \begin{tabular}{l}
$\tilde{0}$ \\
\multirow{N}{0}{} \\
0 \\
0 \\
0
\end{tabular} & $\stackrel{0}{\Xi}$ & 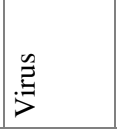 & $\stackrel{0}{\xi}$ \\
\hline 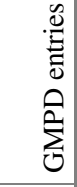 & 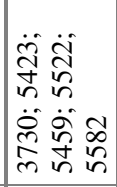 & 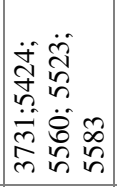 & 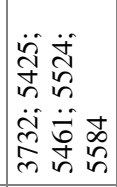 & 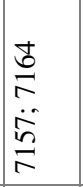 & $\begin{array}{l}\vec{b} \\
i \\
\dot{0} \\
\stackrel{1}{1}\end{array}$ & 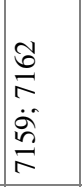 & $\begin{array}{l}\stackrel{n}{n} \\
\ddot{n} \\
\ddot{n} \\
\stackrel{n}{n}\end{array}$ & $\begin{array}{l}n \\
\ddot{n} \\
\ddot{n} \\
\ddot{n} \\
\ddot{n}\end{array}$ & $\begin{array}{l}\ddot{a} \\
\tilde{n} \\
\ddot{n} \\
\ddot{n} \\
\dot{m}\end{array}$ & $\begin{array}{l}\infty \\
0 \\
n \\
n \\
\ddot{0} \\
0 \\
i n\end{array}$ & $\begin{array}{l}0 \\
i n \\
n \\
\dot{0} \\
i n\end{array}$ \\
\hline 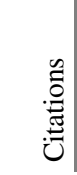 & 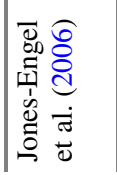 & 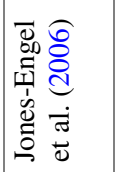 & 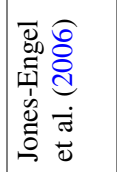 & 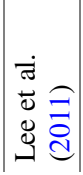 & 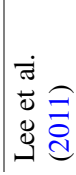 & 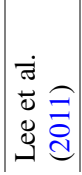 & 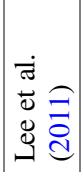 & 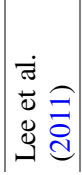 & 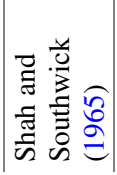 & 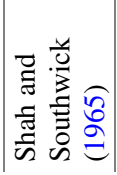 & 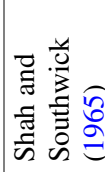 \\
\hline
\end{tabular}


13 Primate Infectious Disease Ecology: Insights and Future Directions at...

\begin{tabular}{|c|c|c|c|c|c|c|c|c|c|}
\hline 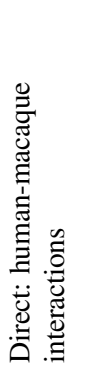 & 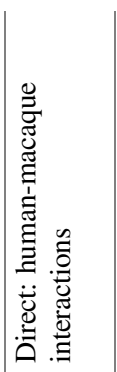 & 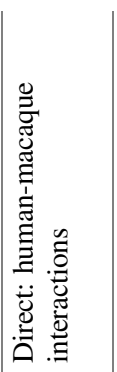 & 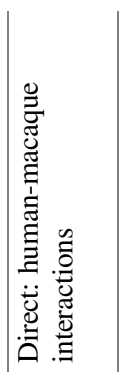 & 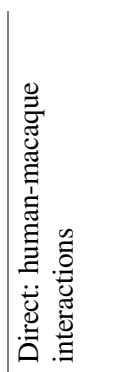 & 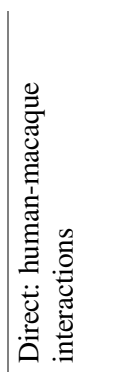 & 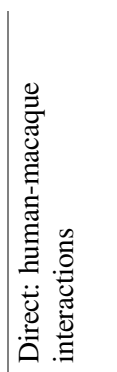 & 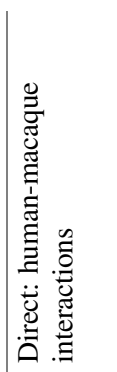 & 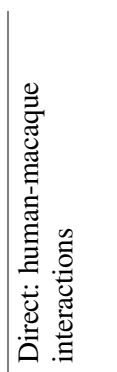 & 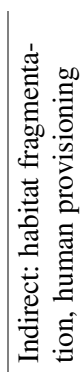 \\
\hline$\frac{m}{0}$ & 8 & 8 & 8 & $\stackrel{8}{8}$ & $\stackrel{1}{2}$ & $\tilde{n}$ & Б․ & f & $\stackrel{5}{0}$ \\
\hline$\infty$ & $\infty$ & $\infty$ & $\triangleright$ & $\infty$ & $\infty$ & $\infty$ & $\triangleright$ & $\infty$ & $\ddot{\theta}$ \\
\hline$\stackrel{\infty}{\sim}$ & $\stackrel{\sim}{\sim}$ & $\stackrel{\infty}{\sim}$ & $\stackrel{\infty}{\sim}$ & $\stackrel{\infty}{\sim}$ & $\stackrel{\infty}{\sim}$ & $\stackrel{\infty}{\sim}$ & $\stackrel{\infty}{\sim}$ & $\stackrel{\sim}{\sim}$ & ֶㅡ \\
\hline 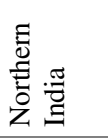 & 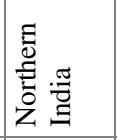 & 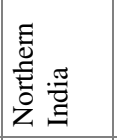 & 泀 & 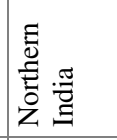 & 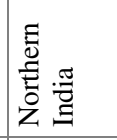 & 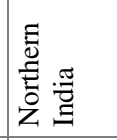 & 胥 & 苞 & 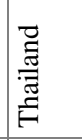 \\
\hline 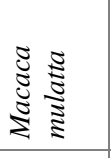 & 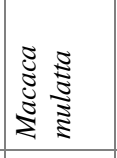 & 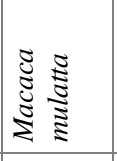 & 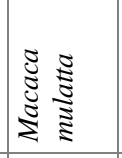 & 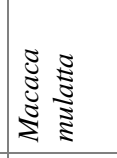 & 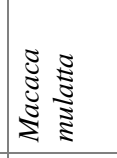 & 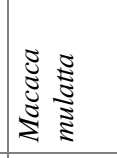 & 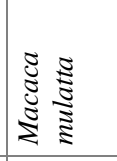 & 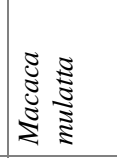 & 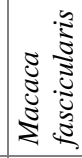 \\
\hline 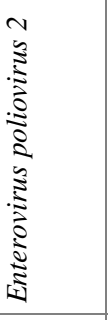 & 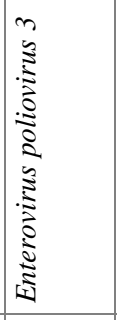 & 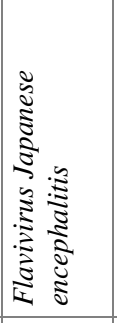 & 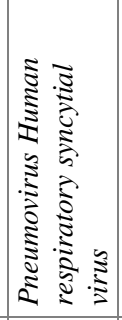 & 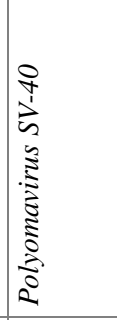 & 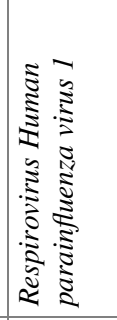 & 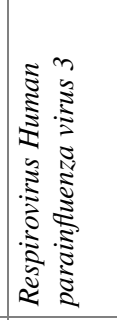 & 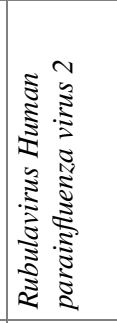 & 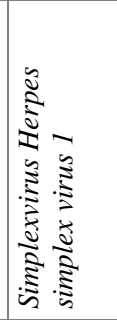 & 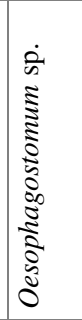 \\
\hline$\stackrel{\varrho}{ٍ}$ & $\stackrel{2}{\equiv}$ & $\stackrel{\mathscr{E}}{\Rightarrow}$ & $\stackrel{\mathscr{\Xi}}{\supset}$ & $\stackrel{\varrho}{\Xi}$ & $\stackrel{\varrho}{\equiv}$ & $\stackrel{0}{\Xi}$ & 忌 & $\stackrel{n}{\Xi}$ & 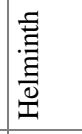 \\
\hline 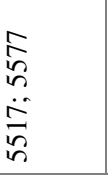 & $\begin{array}{l}\infty \\
i n \\
n \\
\infty \\
\infty \\
n \\
n\end{array}$ & 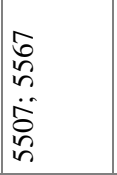 & $\begin{array}{l} \pm \\
i n \\
\ddot{ \pm} \\
\ddot{v} \\
i n\end{array}$ & $\begin{array}{l}2 \\
i n \\
n \\
0 \\
i n\end{array}$ & $\begin{array}{l}\approx \\
n \\
\ddot{n} \\
\ddot{n}\end{array}$ & $\begin{array}{l}m \\
\hat{n} \\
\ddot{n} \\
\dot{n} \\
i n\end{array}$ & $\begin{array}{l}\mathrm{N} \\
i n \\
\ddot{1} \\
i n \\
i n\end{array}$ & $\begin{array}{l}a \\
i n \\
n \\
\ddot{\delta} \\
i n\end{array}$ & 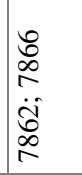 \\
\hline 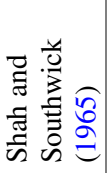 & 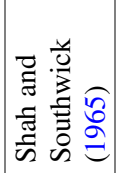 & 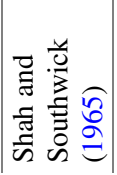 & 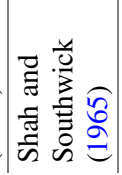 & 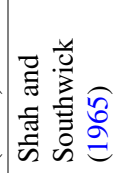 & 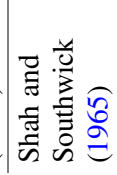 & 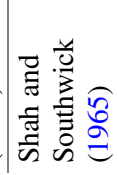 & 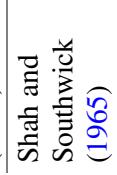 & 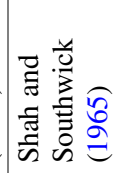 & 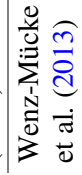 \\
\hline
\end{tabular}




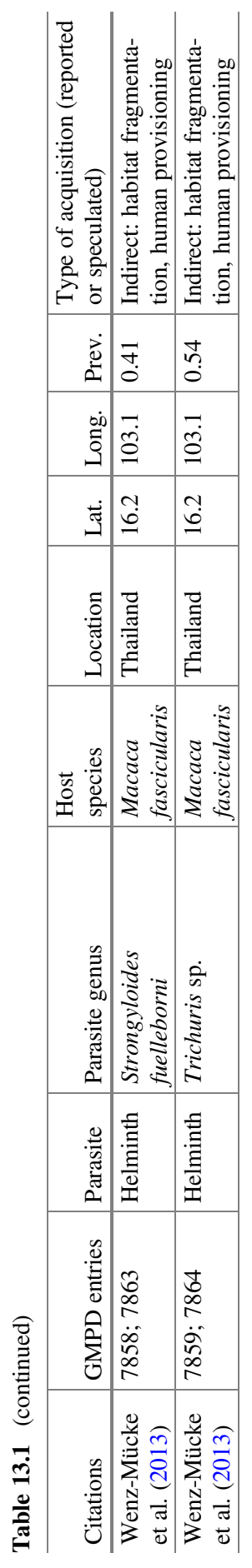


have revealed that anthropogenic factors may indirectly influence their acquisition among macaques [but see Lane et al. (2011) who report a decrease in such acquisition]. In Sri Lanka, for instance, the prevalence of both gastrointestinal protozoan parasites like Cryptosporidium sp., Entamoeba sp., and Balantidium coli and nematodes like Enterobius sp. and Strongyloides sp. was more common among toque macaques in more human-disturbed than pristine environments (Ekanayake et al. 2006). Further, Huffman et al. (2013a) speculate that increased human impact may in part be responsible for why the prevalence of helminth parasites was lower among toque macaques sampled at lower altitudes. Increased contact with anthropogenic landscapes and human-provisioned food was strongly linked to the prevalence of Strongyloides fuelleborni in wild long-tailed macaques in Thailand (Wenz-Mücke et al. 2013). Among populations of critically endangered lion-tailed macaques in Southern India, the anthropogenic fragmentation of their natural habitat was positively correlated to the diversity of gastrointestinal helminths and protozoan parasites (Hussain et al. 2013).

We extracted 10 records (6\% of 161 records) of the protozoan parasite Plasmodium sp., the causative agent of malaria, all from a single study that surveyed wild populations of long-tailed and pig-tailed macaques (Macaca nemestrina) in Borneo (Lee et al. 2011). This study revealed especially high prevalence of three Plasmodium species-P. knowlesi, $P$. cynomolgi, and $P$. inui-among the macaque populations. They also revealed that $P$. knowlesi, previously hypothesized as having been transmitted to humans via anopheline vectors from overlapping macaque populations, was derived from an ancestral malarial parasite that existed before humans came to Southeast Asia. Since then, high prevalence of $P$. knowlesi has been detected among free-living macaques at vegetation mosaics and forest fragments in other parts of Southeast Asia, including Indonesia, Cambodia, Laos, and Vietnam (Huffman et al. 2013b; Zhang et al. 2016). Malaria is now widely recognized as being a threat at human-primate interfaces (Singh et al. 2004). In addition to thriving macaque populations acting as natural reservoirs for these parasites, the fragmented mosaic landscapes of Southeast Asia are also highly conducive to the proliferation of mosquito vector complexes like Anopheles dirus and A. leucosphyrus, which may transmit malaria into otherwise infection-naive macaque and human populations (Moyes et al. 2014).

The least reported type of parasite was bacterial, with only one record speculating that anthropogenic factors may be responsible for the prevalence of Escherichia coli in toque macaques (Huffman et al. 2013a). This was more broadly reflective of the general lack of studies that have focused on the detection of bacterial pathogens in free-living primates (Kaur and Singh 2009; Nunn and Altizer 2006). Bacterial pathogens like Salmonella sp., Shigella sp., and E. coli O157:H7 routinely cause acute diarrheal infection among humans and domestic livestock (Gorski et al. 2011; Rwego et al. 2008; Sinton et al. 2007; Suleyman et al. 2016). They have been previously isolated from wild primate populations in Africa that live in humanperturbed, fragmented habitats (chimpanzees: McLennan et al. 2017; lemurs of Madagascar: Bublitz et al. 2015). Since they strongly overlap and rely heavily on anthropogenic resources, urban and semi-urban macaques may be natural reservoirs 
of these agents, with the potential to disseminate them into overlapping human and critically endangered wildlife populations. A preliminary study at human-rhesus macaque interfaces in Northern India revealed that anthropogenic factors, such as rates of human-macaque aggression and provisioning, were positively correlated with the prevalence of enteric bacteria like Salmonella sp. and E. coli O157:H7 (Beisner et al. 2016). This finding should lead to future assessments of the relative prevalence of enteric bacteria among humans, livestock, and other overlapping macaque populations.

Our GMPD search yielded no studies on parasites in Tibetan macaques, which is somewhat surprising. There is scope for future work to focus on parasite transmission at human-Tibetan macaque interfaces. At both Mt. Emei and Mt. Huangshan, China, where they have been best studied (Zhao 1996; Li 1999), wild Tibetan macaque groups are indeed exposed to anthropogenic factors, particularly tourism. At Mt. Emei, a Buddhist community that is visited by tourists for its temples, there is no regulation of tourist-macaque interactions. Tourists regularly hand-provision the macaques, and there have been reports of tourists suffering fatal injuries from macaque attacks (Zhao 2005). Such intense and frequent contact presents scope for the transmission of parasites. On the other hand, a primate tourism program that is currently in place at Mt. Huangshan restricts the scope for macaque-tourist interactions. This program was laid down following a period between 1994 and 2004, when a group of Tibetan macaques at Mt. Huangshan was "managed" for tourist activity by restricting their home range (Berman et al. 2007). Studies on this group have revealed that intragroup aggression, attacks on infants, and infant mortality rates were all much higher during periods when the group's home range was restricted for tourist viewing than in periods prior to such activity (Berman et al. 2007). Later work in the mid-2000s that was conducted following the period of severe range restriction revealed that the macaques showed increased self-directed behaviors (e.g., self-scratching, yawning, body shake) as well as stress-coping social behaviors (e.g., allogrooming, body contact) when they were closer to tourists, in comparison to when there were no tourists present (reviewed in Matheson et al. 2013). Such tourist activity, now more controlled, may have presented or may continue to present a stressful environment to Tibetan macaques that may heighten the acquisition and transmission of parasites.

\subsection{The Future of Human-Macaque Disease Ecology}

Our review of studies on human-macaque interfaces reveals the detection and confirmation of a range of parasites. Yet many of these studies, based on either symptomatic or mortality-based evidence of pathogenic infection in either humans or macaques, have inferred that disease transmission has occurred without ever having established that transmission did occur (VanderWaal and Ezenwa 2016; VanderWaal et al. 2014). In other words, little or no work has assessed the precise mechanisms and pathways of parasite transmission at human-macaque interfaces. In 
this section, we illustrate how implementing (A) the conceptual frameworks of Coupled Natural and Human Systems and One Health, in combination with (B) cutting-edge network analytical techniques, may significantly enhance our current knowledge of infectious disease transmission at human-macaque interfaces.

\section{(A) Unifying Conceptual Frameworks: Coupled Systems and One Health}

Conflict at human-macaque interfaces may be spatiotemporally variable in form and frequency and may affect parasite transmission in dynamic and sometimes unpredictable ways. In this light, one of the biggest challenges facing research on infectious disease ecology at these, and indeed all human-wildlife interfaces, is the lack of a consensual theoretical or conceptual framework applicable across multiple types of systems.

One framework that may prove useful in this regard stems from the broader conceptualization that human interactions with nature and the environment may be viewed as dynamic, coupled systems (Liu et al. 2007). Since its proposition, the Coupled Natural and Human Systems (or CNHS) approach has presented a significant advancement in our understanding of human impact on abiotic and (more recently) biotic factors. Traditionally, studies examining the interactions between humans and natural phenomena have been largely reductionist in nature (summarized in Liu et al. 2007). They have adopted principles from biology, anthropology, geography, and environmental sciences, with an almost exclusive focus on how a single component of the human system may influence a given property of a natural system or vice versa. Further, they have tended to focus on short-term effects rather than conduct long-term assessments of the feedback effects of such interactions on both human and natural systems. Expanding significantly on these assessments, the CNHS approach explicitly acknowledges that aspects of human systems and natural systems are coupled or interlinked and must therefore be assessed as a collective whole. Multiple, dynamic components of human and natural systems are expected to influence the nature and types of interactions at interfaces, with such impact being expected to reciprocally impact long-term indicators of the overall stability, sustainability, and health of both human populations and natural components.

In its short history, studies implementing CNHS frameworks have primarily focused on the impact of humans on inanimate, abiotic factors (e.g., landscape ecology, climatic conditions: (Foley et al. 2005; Postel et al. 1996) and their longterm effects (e.g., via environmental degradation, natural disasters: Dilley et al. 2005) on human population dynamics and ecology (Liu et al. 2007)). In comparison, fewer studies have tackled the relationship between humans and animate natural systems like wildlife populations (Dickman 2010, 2012). The well-documented nature of interactions and conflict at human-macaque interfaces (reviewed above) offer opportunities to address this gap. Or conversely, the CNHS framework maybe useful to assess the mechanistic processes through which the variant nature of human-macaque interfaces may favor or inhibit the transmission of parasites across human and macaque systems.

As we allude to earlier, macaques may acquire parasites in many ways, including direct physical contact with humans or during social interactions with infected 
conspecifics, changes in foraging strategies induced by anthropogenic landscapes, or human-induced stressors increasing macaques' susceptibility to infection. Implementing the CNHS approach would entail examining the relative likelihood (s) of these mechanisms and indeed whether specific (suites of) attributes of the human system (e.g., community type, history of interactions with macaques, visitors versus tourists) or the macaque system (e.g., age-sex class, group size, speciestypical social style) are linked with the degree to which one type of interface interaction may be expected to prevail over another in influencing parasite acquisition. More tellingly, we reckon that the CNHS framework would finally take research on human-macaque infectious disease research beyond mere descriptions of parasites at interfaces. Expanding on these findings, a CNHS approach would naturally lead to more long-term assessments of the impact of parasite diversity and distribution on indicators of macaque and human population health (e.g., symptomatic evidence for disease outbreaks, stress-induced illness), reproductive success (e.g., the number and fitness of offspring), and survival (e.g., population demographics and infant mortality rates).

Closely related to the CNHS framework is the One Health $(\mathrm{OH})$ concept (derived from the "One Medicine" concept: Schwabe 1984), or the idea that addressing the challenges surrounding human health issues cannot be dissociated from environmental health (or EcoHealth) or from veterinary medical practices associated with treating wild and domestic animals (Destoumieux-Garzon et al. 2018; Zinsstag et al. 2011, 2015). The $\mathrm{OH}$ concept stems from the acknowledgment that the impact of human population expansion on the environment generates negative health outcomes, such as the occurrence of chronic, non-infectious diseases in humans, human and animal exposure to environmental toxins and emerging pollutants like plastics (Kannan et al. 2010; Waters et al. 2016), as well as the emergence of infectious diseases at human-wildlife interfaces (Gomez et al. 2013; Hudson et al. 2002; Nunn et al. 2008; Wolfe et al. 2007). So, it constitutes a global strategy highlighting the need for a holistic, transdisciplinary approach in dealing with the health of humans, animals, and ecosystems (the One Health Initiative).

Since its proposition more than a decade ago, proponents of $\mathrm{OH}$ approaches have (with varying degrees of success) proposed to deal with some of the barriers facing infectious disease research (summarized in Destoumieux-Garzon et al. 2018). We highlight three as being particularly relevant to human-macaque interfaces. The first is a resolution of the extent to which the factors that influence human health outcomes overlap with those that influence the health of natural ecosystems. Human-macaque interfaces are useful to conduct such assessments. This is because many (although not all) social and environmental factors that may potentially drive parasite transmission from humans to macaques, such as direct physical contact, contaminated food or water sources, and the exchange of body fluids, are also likely to transmit agents from macaques to humans (Engel and Jones-Engel 2011; Engel et al. 2008; Jones-Engel et al. 2005; Kaur and Singh 2009).

A second barrier is related to the promotion of interdisciplinary projects that combine veterinary medical assessments to detect and diagnose infectious diseases, with ecological and evolutionary approaches to understand the relationships between 
parasites and their hosts (MacIntosh and Frias 2016; Nesse et al. 2010; Nunn and Altizer 2006). Among all the primates, macaques (particularly rhesus macaques and long-tailed macaques) continue to be the most common genus used in captivity as models for biomedical research (Hannibal et al. 2017; Phillips et al. 2014). Further, as we review above, infectious disease research among free-living macaque populations have had variant foci, ranging from the detection and diagnosis of parasites (Engel and Jones-Engel 2011; Engel et al. 2008; Jones-Engel et al. 2004, 2005), through establishing co-evolutionary links between parasites and macaque hosts (Huffman et al. 2013b), to assessing the social and environmental underpinnings of parasite prevalence and transmission (Duboscq et al. 2016; MacIntosh et al. 2010, 2012; Romano et al. 2016). The OH concept, along with our above-stated argument that human-macaque interfaces are functionally interdependent, coupled systems, would provide a means to bring such diverse foci under a single, unifying framework (Destoumieux-Garzon et al. 2018).

Finally, a third direction involves placing a strong emphasis on complementing strong medical and theoretical knowledge, with current advancements in methodological and data analytical approaches. Below we elaborate on how one set of approaches-Network Analyses - may be especially significant for future research on infectious disease ecology at human-macaque interfaces.

\section{(B) Network-Based Analytical Approaches}

In the last two or three decades, network-based analytical techniques have revolutionized infectious disease epidemiology and ecology (Craft 2015; Craft and Caillaud 2011; Drewe and Perkins 2015; Godfrey 2013; Keeling 2005; Moore and Newman 2000; Newman 2002; VanderWaal and Ezenwa 2016). From a biological perspective, networks are reconstructions of entities (nodes) that are connected to each other based on one or more shared characteristics (edges) (Fig. 13.1a-c). For instance, animal social and spatial networks capture relationships between individuals in a social group linked together based on the frequency with which they interact or the degree of spatial overlap, respectively (reviewed in Brent et al. 2011; Croft et al. 2008; Farine and Whitehead 2015; Kasper and Voelkl 2009; Krause et al. 2007; Lusseau and Newman, 2004; Newman 2004; Sueur et al. 2011b; Wey et al. 2008) (Fig. 13.3a). Bipartite or multimodal networks add a level of complexity by distinguishing two or more components or layers of organization within a system, such that distinctions can be made between the edges that link nodes within the same layer to nodes across layers (Dormann et al. 2017; Kane and Alavi 2008; Latapy et al. 2008) (Fig. 13.3b, c). Third, links based on the degree of genotypic similarity of gastrointestinal microbes isolated from potentially interacting hosts within the same time frame may be used to reconstruct microbial sharing or transmission networks (VanderWaal and Ezenwa 2016; VanderWaal et al. 2014). Finally, in the absence or sparsity of real data, mathematical agent-based models and artificial networks have proven exceptionally useful in modeling the transmission of parasites (Bente et al. 2009; Griffin and Nunn 2012; Nunn 2009; Romano et al. 2016). Such heterogeneity in connectedness within and between the components of socioecological systems may strongly influence the likelihood of parasite acquisition and transmission. 


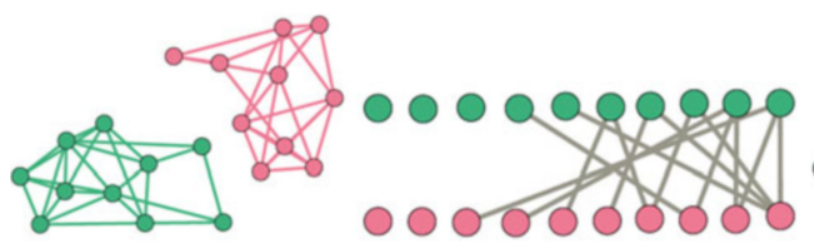

(A)
(B)

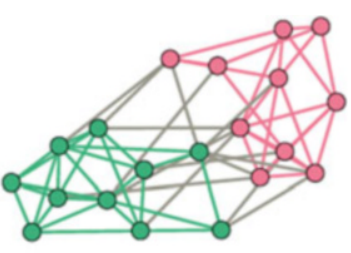

(C)

Fig. 13.3 Hypothetical social (a), bipartite (b), and multimodal (c) networks of the same individuals. Green nodes might represent macaques and pink ones might be humans. Green links are interactions between macaques, pink links are interactions between humans, and gray links are interactions between macaques and humans (interspecies)

Not surprisingly, network approaches, particularly social network analysis, have already found a wide range of applications in infectious disease epidemiology (Craft 2015; Craft and Caillaud 2011; Drewe and Perkins 2015; Godfrey 2013; Keeling 2005; VanderWaal and Ezenwa 2016). We briefly review these applications and related studies below. Recognizing the relative dearth in the implementation of network approaches at human-macaque interfaces, we also highlight some context (s) in which they may be implemented in human-macaque infectious disease ecological research.

Social Networks and Parasite Acquisition In humans and other animals, heterogeneity in space use overlap or contact social behavior may strongly influence the acquisition of parasites (reviewed in Drewe and Perkins 2015; Kappeler et al. 2015; Silk et al. 2017). Such heterogeneity can be modeled using social network analysis (Brent et al. 2011; Croft et al. 2008; Farine and Whitehead 2015; Krause et al. 2007; Sueur et al. 2011b). The first applications of social network approaches in the context of disease transmission were focused on humans, particularly in the spread of sexually transmitted diseases (or STDs) (Klovdahl 1985) and later following the detection of the severe acute respiratory syndrome (SARS) outbreak in 2003 (Meyers 2007). Since then, social networks across a wide range of taxa have been used to identify central or well-connected individuals, which may be potential "super spreaders" of parasites [e.g., lizards (Egernia stokesii): Godfrey et al. 2009; spider monkeys (Ateles geoffroyi): Rimbach et al. 2015; meerkats (Suricata suricatta): Drewe 2010; Japanese macaques: MacIntosh et al. 2012; reviewed in Drewe and Perkins 2015; VanderWaal and Ezenwa 2016]. In wild Japanese macaques, for instance, high-ranking individuals with more direct and indirect connections, or eigenvector centrality (Newman 2006) in their social grooming networks, were also shown to have greater species richness and infection intensities of nematode parasites (MacIntosh et al. 2012). Yet having strong and diverse social connections, rather than increasing parasite acquisition owing to contact-mediated transmission, may actually decrease the likelihood of such acquisition by mitigating stressors or enhancing immune function (e.g., Balasubramaniam et al. 2016; Cohen et al. 2015; 
Hennessy et al. 2009). Consistent with this "social buffering hypothesis," work on captive rhesus macaques revealed that individuals with the strongest and most diverse social grooming and huddling connections were also the least prone to the acquisition of environmental bacterial pathogens (Balasubramaniam et al. 2016). Finally, aside from inter-individual differences in contact patterns, the higher order structure of social networks may also influence parasite transmission in contrasting ways. For instance, increased community modularity or substructuring in social networks, on account of individuals interacting more with subsets of preferred partners (Fushing et al. 2013; Newman 2006; Whitehead and Dufault 1999), may enhance parasite transmission within subgroups while presenting "social bottlenecks" to the group-wide spread of parasites (Griffin and Nunn 2012; Huang and Li 2007; Nunn et al. 2011, 2015; Romano et al. 2018; Salathe and Jones 2010). On the other hand, dense, well-connected networks, with a higher efficiency (the inverse of the number of shortest paths in the network), may facilitate rather than hinder the rapid transmission of parasites (Drewe and Perkins 2015; Griffin and Nunn 2012; Pasquaretta et al. 2014).

Such dynamic relationships between social networks and parasite acquisition suggest that broader socioecological contexts may determine the circumstances under which social life may be beneficial versus detrimental to infectious disease risk. The variant nature of the human-macaque interface may present such contexts and thereby influence parasite acquisition by altering the structure and connectedness of macaque social networks. For instance, higher frequencies of interactions with humans and/or changes to macaque movement or foraging behavior in landscapes altered by anthropogenic disturbance, by constraining the time available for macaques to engage in social interactions (Dunbar 1992; Kaburu et al. 2019; Marty et al. 2019), may lead to more modular, substructured social networks which may present bottlenecks to parasite transmission. On the other hand, such interactions or anthropogenic changes may present environmental stressors to the macaques (e.g., Barbary macaques: Carne et al. 2017; Marechal et al. 2011, 2016), in which individuals possessing strong and diverse social networks may benefit by being socially buffered against infection. In summary, future research may focus on establishing the precise mechanism(s) of super spreading, social bottlenecking, or stress-induced acquisition of parasites, through which social or contact network connectedness may influence parasite transmission dynamics at human-macaque interfaces.

Bipartite and Multimodal Networks Social networks have proven exceptionally useful to model the acquisition and transmission of parasites. Yet by themselves, they are somewhat limited in not capturing heterogeneity at higher organizational scales, for instance, across interactions between different components of a social or ecological system. This may be the reason why most epidemiological studies implementing network approaches to model heterogeneity in contact patterns have focused on either human systems or more recently wildlife systems (reviewed above), but almost never at the human-wildlife interface. Bipartite and multimodal networks, which establish connections between different interlinked components of 
a system, may prove especially useful in this regard (Dormann et al. 2017; Kane and Alavi 2008; Latapy et al. 2008; Finn et al. 2019). Recently, bipartite networks are beginning to feature in ecological and evolutionary research (reviewed in Bascompte and Jordano 2014; Cagnolo et al. 2011; Dormann et al. 2017), as illustrated by their being used to model marine food webs (Rezende et al. 2009), mutualistic interactions between flowers and seed-dispersing animal pollinators (Spiesman and Gratton 2016; Stang et al. 2009; Vazquez et al. 2009), and, more pertinently, host-parasitoid relationships (Laliberte and Tylianakis 2010; Poulin et al. 2013). For networks that combine links both within and across system components, some researchers have coined the term "multimodal networks," aka "multilayer" or "multislice networks" (Kane and Alavi 2008; Finn et al. 2019).

Their potential to model the connections of complex systems make bipartite and multimodal networks highly relevant tools for infectious disease research at humanwildlife interfaces. Yet to our knowledge, they have not been extensively used in this context. In the context of primates and EIDs, Gomez et al. (2013) used a combination of bipartite network construction and social network analytical tools to identify primate species that are likely to harbor EIDs. They constructed a bipartite network that linked each primate species with each parasite isolated from them. From this, they projected a unipartite "social" network in which primate species as nodes were linked to each other by edges weighted by the number of parasites they shared. They then revealed that species that were highly central in this "primate-parasite" network were also the most likely to function as "super spreaders" of EIDs to humans or at least most likely to share those EIDs with humans, suggesting potential conservation implications as well. However, only one macaque species- the toque macaquewas among the top 10 most central primates in this network. So, the extent to which macaques pose threats as transmitters of EIDs remains unclear [and more generally reflective of a knowledge gap in infectious disease research in East and Southeast Asia (Hopkins and Nunn 2007)], though this is undoubtedly likely to vary across contexts.

As reviewed in the previous section, humans and macaques engage in a variety of interactions at interfaces, some forms of which have already been linked to the acquisition and transmission of parasites (summarized in Table 13.1). To assess the mechanistic bases of such transmission, we recommend that future studies, implementing a CNHS approach, focus on constructing bipartite and multimodal networks based on intra- and interspecies interactions and spatial overlap at humanmacaque interfaces. For such networks, the choice of system components may also be informed by the characteristics of the parasite studied (Craft 2015). For instance, the transmission of RNA respiratory and retroviruses between humans and macaques may require intense contact events such as bites and scratches and the exchange of body fluids like blood and saliva. Yet their fast mutation rates and short generation times may make them difficult to detect. So, multimodal networks of mild and severe contact aggressive interactions among and between humans and macaques over shorter durations of time may be useful for these purposes. On the other hand, the transmission of gastrointestinal helminths, protozoa, and enteric bacteria may require just subtle interactions such as human provisioning of 
macaques, acquisition from soil and anthropogenic surfaces, and macaque social grooming and contact huddling interactions, any or all of which would involve fecaloral transmission (Balasubramaniam et al. 2016; Beisner et al. 2014, 2016; MacIntosh et al. 2012). Given that enteric bacteria may also survive longer in anthropogenic environments such as human-contaminated food, water sources and substrates (Sinton et al. 2007), moist soil (Kilonzo et al. 2011), and livestock (Craft 2015; Rwego et al. 2007; VanderWaal et al. 2014), unraveling their transmission routes may involve the construction of "multipartite" networks connecting contact patterns and spatial overlap between these biotic and abiotic factors. An even higher level of complexity may be required to detect the transmission routes of vector-borne malarial parasites like Plasmodium knowlesi (Abkallo et al. 2014; Huffman et al. 2013b; Lee et al. 2011). These may depend heavily on geospatial variation in the distribution and overlap of humans, reservoir macaques, and other host wildlife populations, anopheline vectors, and a host of environmental factors that may be conducive to the completion of both vector and pathogen life histories (Lee et al. 2011; Moyes et al. 2014).

Microbial Transmission Networks More recently, the phylogenetic relationships of symbiotic gut microbes isolated from animal hosts have been used to construct microbial transmission networks (VanderWaal and Ezenwa 2016). Such networks offer special advantages to detecting the potential transmission pathways of parasites that spread through the fecal-oral route (Sears et al. 1950, 1956; Tenaillon et al. 2010). Symbiotic microbes like gastrointestinal E. coli are present in almost every individual, have shared evolutionary histories with intestinal pathogens, and are typically acquired via fecal-oral routes (Caugant et al. 1981; Sears et al. 1950, 1956). So, if two individuals have genotypically similar or identical strains of $E$. coli, they are likely to have either shared the strain via fecal-oral transmission, which may occur either through direct social contact or through using a common environmental source (Chiyo et al. 2014; Springer et al. 2016; VanderWaal et al. 2013, 2014). Further, they rarely (if ever) alter the behavior of the host (VanderWaal et al. 2014), which allows researchers to potentially detect subtle transmission events that may signal the potential for a more devastating outbreak. Limited research to date has revealed strong links between the degree of dyadic similarity in E. coli and the frequency of animal space use overlap and/or social contact patterns (Balasubramaniam et al. 2018; Chiyo et al. 2014; Springer et al. 2016; VanderWaal et al. 2013, 2014). Recently, a study on captive rhesus macaques established that macaques in the same social network communities were more likely to share strains of $E$. coli among themselves than they were to macaques from other communities (Balasubramaniam et al. 2018). Previous assessments at human-primate interfaces have revealed that the population genetic structure of gut $E$. coli from great ape populations living in human-perturbed habitats was more similar to E. coli from humans and livestock than they were to bacteria. Such findings encourage future efforts to construct microbial transmission networks between humans and overlapping macaque populations, which may serve as models to gauge the potential transmission pathways of parasites with greater precision. However, the lack of 
discernible mortality or sickness behaviors associated with acquiring non-pathogenic microbes makes them models, rather than accurate forecasters, for parasite transmission.

Agent-Based Modeling When data on biological systems are either unavailable or incomplete, mathematical models offer ways of dealing with such inadequacies. In simple terms, a model may be thought of as a simplified version of a study system used to better understand it (Epstein and Axtell 1996; Minsky 1965). Thus, the complexity of a model has to be lower than that of the study system; otherwise its usefulness is lost. The main advantage of a computational model is that it can be tested infinitely by recreating or simulating a situation in the same way many (thousands of) times by adding, removing, or varying parameters and measuring emergent characteristics. These results are then usually compared with findings from empirical data to confirm or reject the tested hypotheses and accept (at least temporarily) the one for which the simulations best explain the data (Epstein and Axtell 1996; Minsky 1965). Furthermore, outcomes from these models may reveal complex or unexpected effects which may be different from, or even go undetected, based on a priori predictions. This might in turn provide a stronger basis to make other predictions, including those related to the relative role(s) of different predictive factors, in future assessments of biological systems (Epstein and Axtell 1996; Minsky 1965).

Unsurprisingly, the bottom-up approaches of simulated models have made them exceptionally useful tools to understand the acquisition and transmission of parasites. The first mathematical models of parasite transmission did not implement social network approaches: virtual individuals moved and interacted randomly in their environments (Wilensky and Stroup 1999). From individual characteristics (e.g., age, sex, hierarchical rank) and basic interaction rules (social contact, spatial proximity, conflicts, etc.), these tried to assess how the more global phenomena of parasite prevalence and outbreak potential emerge in a system (Romano et al. 2016; Rushmore et al. 2014). In these models, only the $\mathrm{R}_{0}$, the initial number of infected agents and their interaction rates, mattered. $R_{0}$ is the basic reproduction number used to quantify the transmission potential of a parasite, defined as the number of secondary infections caused by a single infected individual introduced into a population made up entirely of susceptible individuals. However, following the acknowledgment that individuals do not move or interact randomly, social networks have been integrated into these models during the last decade or so (Griffin and Nunn 2012; Huang and Li 2007; McCabe and Nunn 2018; Nunn 2009).

Expanding beyond homogenous populations that contain only susceptible individuals that interact randomly, recent studies have integrated network approaches with a classic set of individual-based models used in epidemiology that classify individuals or "agents" into moving between susceptible infected and resistant (or SIR) classes or compartments (Bansal et al. 2007; Brauer 2008; Grimm and Railsback 2005; Kohler and Gumerman 2000). Such integrated network-based SIR models now form the basis of many epidemiological assessments in primate systems (Griffin and Nunn 2012; Kohler and Gumerman 2000; McCabe and Nunn, 2018; 
Rushmore et al. 2014). For instance, the likelihood of parasite transmission from A to B may be a function of (1) whether A is already infected, (2) the likelihood of a link between A and B in their social network, and (3) the per-contact transmission probability "Beta." Simulations are run until set criteria are reached, e.g., either the extinction or saturation of infection throughout the group, at which time the average outbreak size or $R_{\text {infinity }}$ is calculated (Diekmann et al. 1998; Keeling 1999). The implemented social network might be theoretical (Sueur et al. 2012), based on empirical data (Romano et al. 2016; Rushmore et al. 2014), or both (Griffin and Nunn 2012) depending on the aims of the study.

In a comparative evolutionary analysis that used both natural primate datasets and simulated networks, Griffin and Nunn (2012) used an SIR model to reveal how increased community modularity in the social network, despite a larger group size, negatively impacts parasite success. In a more applied example of the implementation of agent-based models, Rushmore et al. (2014) modeled the social network of a wild population of chimpanzees in order to target specific individuals to vaccinate. Based on their social centrality, they revealed that it was sufficient to target fewer, more central individuals for the same result regarding controlling the spread of infection. In this study, social network position was a model parameter, but it might also be the study object if the model includes feedback loops between the social network and parasite acquisition. Corner et al. (2003), for instance, not only showed that social networks of Australian possums (Trichosurus vulpecula) have an effect on the transmission rate of tuberculosis (TB) but also that the spread of TB had a feedback effect on the social network: infected possums showed higher proximity degree and betweenness centrality than non-infected possums. To our knowledge, agent-based models have not been applied to assess parasite transmission at humanmacaque interfaces. In order to do so, we recommend that future research implement the SIR approach, but focus on using multimodal network models in the place of social networks which may account for an added level of complexity to model the heterogeneity between human-macaque and macaque-macaque interactions.

\subsection{Conclusions}

Our goals in writing this chapter were threefold. First, we wanted to convey how, despite a recent surge in primate infectious disease ecological research during the last two decades, relatively few efforts have focused on the impact of humans and anthropogenic factors on disease risk in wild primates. Second, we hope to have illustrated how our current knowledge of human-macaque interactions in particular present "starting points" from which long-term, in-depth assessments of the ecology of parasite acquisition and transmission at human-primate interfaces may be conducted. We believe that such efforts would need to integrate ethnoprimatology and infectious disease ecology in order to be successful. Finally, we hope to have convinced readers of how research on infectious disease ecology at human-macaque interfaces are also in need of the implementation of both novel conceptual 
frameworks (e.g., One Health, Coupled Systems) and cutting-edge analytical approaches (e.g., Network Analyses, mathematical modeling). Such approaches not only bolster the scope of epidemiological research but are also imperative for the conservation and management of both threatened and potentially problematic free-living primate (and indeed other wildlife) populations.

\section{References}

Abkallo HM, Liu W, Hokama S, Ferreira PE, Nakazawa S, Maeno Y, Quang NT, Kobayashi N, Kaneko O, Huffman MA, Kawai S, Marchand RP, Carter R, Hahn BH, Culleton R (2014) DNA from pre-erythrocytic stage malaria parasites is detectable by PCR in the faeces and blood of hosts. Int J Parasitol 14:467-473

Amoroso CR, Frink AG, Nunn CL (2017) Water choice as a counterstrategy to faecally transmitted disease: an experimental study in captive lemurs. Behaviour 154:1239-1258

An L, Lopez-Carr D (2012) Understanding human decisions in coupled natural and human systems. Ecol Model 229:1-4

Balasubramaniam KN, Dittmar K, Berman CM, Butovskaya M, Cooper MA, Majolo B, Ogawa H, Schino G, Thierry B, de Waal FBM (2012) Hierarchical steepness, counter-aggression, and macaque social style scale. Am J Primatol 74(10):915-925

Balasubramaniam KN, Beisner BA, Vandeleest J, Atwill ER, McCowan B (2016) Social buffering and contact transmission: network connections have beneficial and detrimental effects on Shigella infection risk among captive rhesus macaques. PeerJ 4:e2630

Balasubramaniam KN, Beisner BA, Guan J, Vandeleest J, Fushing H, Atwill ER, McCowan B (2018) Social network community structure is associated with the sharing of commensal E. coli among captive rhesus macaques (Macaca mulatta). PeerJ 6:e4271

Bansal S, Grenfell BT, Meyers LA (2007) When individual behaviour matters: homogeneous and network models in epidemiology. J R Soc Interface 4:879-891

Barua M, Bhagwat SA, Jadhav S (2013) The hidden dimensions of human-wildlife conflict: health impacts, opportunity and transaction costs. Biol Conserv 157:309-316

Bascompte J, Jordano P (2014) Mutualistic networks. Princeton University Press, Princeton, NJ

Beisner BA, Heagarty A, Seil S, Balasubramaniam KN, Atwill ER, Gupta BK, Tyagi PC, Chauhan NPS, Bonal BS, Sinha PR, McCowan B (2014) Human-wildlife conflict: proximate predictors of aggression between humans and rhesus macaques in India. Primates 57:459-469

Beisner BA, Balasubramaniam KN, Fernandez K, Heagerty A, Seil SK, Atwill ER, Gupta BK, Tyagi PC, Chauhan PS, Bonal BS, Sinha PR, McCowan B (2016) Prevalence of enteric bacterial parasites with respect to anthropogenic factors among commensal rhesus macaques in Dehradun, India. Primates 57:459-469

Bente D, Gren J, Strong JE, Feldmann H (2009) Disease modeling for ebola and marburg viruses. Dis Model Mech 2:12-17

Berman CM, Li J, Ogawa H, Ionica C, Yin H (2007) Primate tourism, range restriction, and infant risk among Macaca thibetana at Mt. Huangshan, China. Int J Primatol 28:1123-1141

Brauer F (2008) Compartmental models in epidemiology, chapter 2. In: Brauer F, van den Driessche P, Wu J (eds) Mathematical epidemiology. Springer, Berlin

Brent LJN, Lehmann J, Ramos-Fernandez G (2011) Social network analysis in the study of nonhuman primates: a historical perspective. Am J Primatol 73(8):720-730

Bublitz DC, Wright PC, Rasambainarivo FT, Arrigo-nelson SJ, Bodager JR, Gillespie TR (2015) Pathogenic enterobacteria in lemurs associated with anthropogenic disturbance. Am J Primatol 77:330-337

Cagnolo L, Salvo A, Valladares G (2011) Network topology: patterns and mechanisms in plantherbivore and host-parasitoid food webs. J Anim Ecol 80:342-351 
Carne C, Semple S, MacLarnon A, Majolo B, Marechal L (2017) Implications of tourist-macaque interactions for disease transmission. EcoHealth 14:704-717

Caugant DA, Levin BR, Selander RK (1981) Genetic diversity and temporal variation in the E. coli population of a human host. Genetics $98: 467-490$

Chapman CA, Gillespie TR, Goldberg T (2005) Primates and the ecology of their infectious diseases: how will anthropogenic change affect host-parasite interactions? Evol Anthropol 14:134-144

Chapman CA, Speirs ML, Gillespie TR, Holland T, Austad KM (2006a) Life on the edge: gastrointestinal parasites from the forest edge and interior primate groups. Am J Primatol 68:397-409

Chapman CA, Wasserman MD, Gillespie TR, Speirs ML, Lawes MJ, Saj TL et al (2006b) Do food availability, parasitism, and stress have synergistic effects on red colobus populations living in forest fragments? Am J Phys Anthropol 131:525-534

Chapman CA, Rothman JM, Hodder SAM (2009) Can parasite infection be a selective force influencing primate group size? A test with red colobus. In: Huffman MA, Chapman CA (eds) Primate parasite ecology. Cambridge University Press, Cambridge, pp 423-440

Chiyo PI, Grieneisen LE, Wittemyer G, Moss CJ, Lee PC, Douglas-hamilton I, Archie EA (2014) The influence of social structure, habitat, and host traits on the transmission of Escherichia coli in wild elephants. PLoS One 9:e93408

Cohen S, Janicki-Deverts D, Turner RB, Doyle WJ (2015) Does hugging provide stress-buffering social support? a study of susceptibility to upper respiratory infection and illness. Psychol Sci 26:135-147

Cords M (2013) The behavior, ecology, and social evolution of Cercopithecine monkeys. In: Mitani JC, Call J, Kappeler PM, Palombit RA, Silk JB (eds) The evolution of primate societies. University of Chicago Press, Chicago, pp 91-112

Corner LAL, Pfeiffer DU, Morris RS (2003) Social-network analysis of Mycobacterium bovis transmission among captive brushtail possums (Trichosurus vulpecula). Prev Vet Med 59:147-167

Craft ME (2015) Infectious disease transmission and contact networks in wildlife and livestock. Philos Trans R Soc Lond B Biol Sci 370:1-12

Craft ME, Caillaud D (2011) Network models: an underutilized tool in wildlife epidemiology. Interdiscip Perspect Infect Dis 2011:1-12

Croft DP, James R, Krause J (2008) Exploring animal social networks. Princeton University Press, Princeton, NJ

Destoumieux-Garzon D, Mavingui P, Boetsch G, Biossier J, Darriet F, Duboz P, Fritsch C, Giradoux P, Le Roux P, Morand S, Paillard C, Pontier D, Sueur C, Voituron Y (2018) The one health concept: 10 years old and a long road ahead. Front Vet Sci 5:14

Dickman AJ (2010) Complexities of conflict: the importance of considering social factors for effectively resolving human-wildlife conflict. Anim Conserv 13:458-466

Dickman AJ (2012) From cheetahs to chimpanzees: a comparative review of the drivers of humancarnivore conflict and human-primate conflict. Folia Primatol 83:377-387

Diekmann O, De Jong MCM, Metz JJ (1998) A deterministic epidemic model taking account of repeated contacts between the same individuals. J Appl Probab 35:448-462

Dilley M, Chen SR, Deichmann U, Lerner-Lam LA, Arnold M, Agwe J, Buys P, Kjekstad O, Lyon B, Yetman G (2005) Natural disaster hotspots: a global risk analysis. In: Bank W (ed) Disaster risk management. World Bank and Columbia University, Washington, DC

Dore KM, Riley EP, Fuentes A (2017) Ethnoprimatology: a practical guide to research on the human-nonhuman primate interface. Cambridge University Press, Cambridge

Dormann C, Frund J, Schaefer MH (2017) Identifying causes of patterns in ecological networks: opportunities and limitations. Annu Rev Ecol Evol Syst 48:559-584

Drewe JA (2010) Who infects whom? Social networks and tuberculosis transmission in wild meerkats. Proc Biol Sci 277:633-642 
Drewe JA, Perkins SE (2015) Disease transmission in animal social networks. In: Krause J, James R, Franks DW, Croft DP (eds) Animal social networks. Oxford University Press, Oxford, pp $95-110$

Duboscq J, Romano V, Sueur C, MacIntosh AJJ (2016) Network centrality and seasonality interact to predict lice load in a social primate. Sci Rep 6:22095

Dunbar RIM (1992) Time: a hidden constraint on the behavioural ecology of baboons. Behav Ecol Sociobiol 31:35-49

Ekanayake DK, Arulkanthan A, Horadagoda NU, Sanjeevani M, Kieft R, Gunatilake S, Dittus WP (2006) Prevalence of cryptosporidium and other enteric parasites among wild non-human primates in Polonnaruwa, Sri Lanka. Am J Trop Med Hyg 74:322-329

Engel GA, Jones-Engel L (2011) The role of Macaca fascicularis in infectious disease transmission. In: Gumert MD, Fuentes A, Jones-Engel L (eds) Monkeys on the edge: ecology and management of long-tailed macaques and their interface with humans. Cambridge University Press, Cambridge, pp 183-203

Engel GA, Pizarro M, Shaw E, Cortes J, Fuentes A, Barry P, Lerche N, Grant R, Cohn D, JonesEngel L (2008) Unique pattern of enzootic primate viruses in Gibralter macaques. Emerg Infect Dis 14:1112-1115

Epstein J, Axtell R (1996) Growing artificial societies: social science from the bottom Up. MIT Press, Cambridge, MA

Farine DR, Whitehead H (2015) Constructing, conducting and interpreting animal social network analysis. J Anim Ecol 84:1144-1163

Feeroz MM, Soliven K, Small CT, Engel GA, Pacheco MA, Yee JL, Wang X, Kamrul Hasan M, Oh G, Levine KL, Rabiul Alam SM, Craig KL, Jackson DL, Lee EG, Barry PA, Lerche NW, Escalante AA, Matsen Iv FA, Linial ML, Jones-Engel L (2013) Population dynamics of rhesus macaques and associated foamy virus in Bangladesh. Emerg Microbes Infect 2:e29

Fehlmann G, O’Riain MJ, Kerr-Smith C, King AJ (2016) Adaptive space use by baboons (Papio ursinus) in response to management interventions in a human-changed landscape. Anim Conserv 20:101-109

Fiennes R (1967) Zoonoses of primates. Cornell University Press, Ithaca, NY

Finn KR, Silk MJ, Porter MA, Pinter-Wollman N (2019) The use of multilayer network analysis in animal behaviour. Anim Behav 149:7-22

Foley JA, DeFries R, Asner GP, Barford C, Bonan G, Carpenter SR, Chapin FS, Coe MT, Daily GC, Gibbs HK, Helkowski JH, Holloway T, Howard EA, Kucharik CJ, Monfreda C, Patz JA, Prentice IC, Ramankutty N, Snyder PK (2005) Global consequences of land use. Science 309 (5734):570-574

Frias L, MacIntosh AJJ (2018) Threatened hosts, threatened parasites? Parasite diversity and distribution in Red-Listed Primates. In: Behie AM, Teichroeb J, Malone N (eds) Primate research and conservation in the anthropocene. Cambridge University Press, Cambridge

Fuentes A (2006) Patterns and context of human-macaque interactions in Gibraltar. In: Hodges JK, Cortes J (eds) The Barbary macaque: biology, management, and conservation. Nottingham University Press, Nottingham, pp 169-184

Fuentes A (2012) Ethnoprimatology and the anthropology of the human-primate interface. Annu Rev Anthropol 41:101-117

Fuentes A, Hockings KJ (2010) The ethnoprimatological approach in primatology. Am J Primatol 72(10):841-847

Fuentes A, Rompis ALT, Arta Putra IGA, Watiniasih NL, Nyoman Suartha I, Wandia IN et al (2011) Macaque behavior at the human-monkey interface: the activity and demography of semifree-ranging Macaca fascicularis at Padangtegal, Bali, Indonesia. In: Gumert MD, Fuentes A, Jones-Engel L (eds) Monkeys on the edge: ecology and management of long-tailed macaques and their interface with humans. Cambridge University Press, Cambridge, pp 159-179

Fushing H, Wang H, VanderWaal K, McCowan B, Koehl P (2013) Multi-scale clustering by building a robust and self correcting ultrametric topology on data points. PLoS One 8(2):e56259 
Gillespie TR, Chapman CA, Greiner EC (2005) Effects of logging on gastrointestinal parasite infections and infection risk in African primates. J Appl Ecol 42:699-707

Godfrey SS (2013) Networks and the ecology of parasite transmission: a framework for wildlife parasitology. Int J Parasitol Parasites Wildl 2:235-245

Godfrey SS, Bull CM, James R, Murray K (2009) Network structure and parasite transmission in a group-living lizard, the gidgee skink, Egernia stokesii. Behav Ecol Sociobiol 63:1045-1056

Goldberg T, Gillespie TR, Rwego IB, Wheeler E, Estoff EL, Chapman CA (2007) Patterns of gastrointestinal bacterial exchange between chimpanzees and humans involved in research and tourism in western Uganda. Biol Conserv 135:511-517

Gomez JM, Nunn CL, Verdu M (2013) Centrality in primate-parasite networks reveals the potential for the transmission of emerging infectious diseases to humans. PNAS 110:7738-7741

Gorski L, Parker CT, Liang A, Cooley MB, Jay-Russell MT, Gordus AG et al (2011) Prevalence, distribution, and diversity of Salmonella enterica in a major produce region of California. Appl Environ Microbiol 77(8):2734-2748

Grenfell BT, Dobson AP (1995) Ecology of infectious diseases in natural populations. Cambridge University Press, Cambridge

Griffin RH, Nunn CL (2012) Community structure and the spread of infectious disease in primate social networks. Evol Ecol 26(4):779-800

Grimm V, Railsback SF (2005) Individual based modeling in ecology. Princeton University Press, Princeton, NJ

Gumert MD, Fuentes A, Jones-Engel L (eds) (2011) Monkeys on the edge: ecology and management of long-tailed macaques and their interface with humans. Cambridge University Press, Cambridge

Hannibal DL, Bliss-Moreau E, Vandeleest J, McCowan B, Capitanio J (2017) Laboratory rhesus macaque social housing and social changes: implications for research. Am J Primatol 79:1-14

Hennessy MB, Kaiser S, Sachser N (2009) Social buffering of the stress response: diversity, mechanisms, and functions. Front Neuroendocrinol 30:470-482

Hinde RA (1976) Interactions, relationships and social structure. Man 11:1-17

Hockings KJ, Anderson JR, Matsuzawa T (2012) Socioecological adaptations by chimpanzees (Pan troglodytes) verus inhabiting an anthropogenically impacted habitat. Anim Behav 83:801-810

Hoffman TS, O'Riain MJ (2012) Monkey management: using spatial ecology to understand the extent and severity of human-baboon conflict in the Cape Peninsula, South Africa. Ecol Soc 17:13

Hopkins ME, Nunn CL (2007) A global gap analysis of infectious agents in wild primates. Divers Distrib 13:561-572

Huang W, Li C (2007) Epidemic spreading in scale-free networks with community structure. J Stat Mech 2007:P01014

Hudson PJ, Rizzoli A, Grenfell BT, Heesterbeek H, Dobson AP (2002) The ecology of wildlife diseases. Oxford University Press, New York

Huffman MA (2016) Primate self-medication, passive prevention and active treatment-a brief review. Int J Multidiscip Stud 3:1-10

Huffman MA, Chapman CA (2009) Primate parasite ecology: the dynamics and study of hostparasite relationships. Cambridge University Press, Cambridge

Huffman MA, Nahallage CAD, Hasegawa H, Ekanayake S, De Silva LDGG, Athauda IRK (2013a) Preliminary survey of the distribution of four potentially zoonotic parasite species among primates in Sri Lanka. J Natl Sci Found 41:319-326

Huffman MA, Satou M, Kawai S, Maeno Y (2013b) New perspectives on the transmission of malaria between macaques and humans: the case of Vietnam. Folia Primatol 84:288-289

Hussain S, Ram MS, Kumar A, Shivaji S, Umapathy G (2013) Human presence increases parasitic load in endangered lion-tailed macaques (Macaca silenus) in its fragmented rainforest habitats in southern India. PLoS One 8:e63685 
IUCN (2019) The IUCN Red list of threatened species. Version 2019-2. http://www.iucnredlist.org. Accessed 18 July 2019

Jaman MF, Huffman MA (2013) The effect of urban and rural habitats and resource type on activity budgets of commensal rhesus macaques (Macaca mulatta) in Bangladesh. Primates 54:49-59

Jones-Engel L, Engel GA, Schillaci MA, Kyes K, Froehlich J, Paputungan U, Kyes RC (2004) Prevalence of enteric parasites in pet macaques in Sulawesi, Indonesia. Am J Primatol 62:71-82

Jones-Engel L, Engel GA, Schillaci MA, Rompis ALT, Putra A, Suaryana KG, Fuentes A, Beer B, Hicks S, White R, Wilson B, Allan JS (2005) Primate to human retroviral transmission in Asia. Emerg Infect Dis 7:1028-1035

Jones-Engel L, Engel GA, Heidrich J, Chalise M, Poudel N, Viscidi R, Barry PA, Allan JS, Grant R, Kyes R (2006) Temple monkeys and health implications of commensalism, Kathmandu, Nepal. Emerg Infect Dis 12:900-906

Kaburu SSK, Marty PR, Beisner B, Balasubramaniam KN, Bliss-Moreau E, Kaur K, Mohan L, McCowan B (2019) Rates of human-macaque interactions affect grooming behavior among urban-dwelling rhesus macaques (Macaca mulatta). Am J Phys Anthropol 168:92-103

Kane GC, Alavi M (2008) Casting the net: a multimodal network perspective on user-system interactions. Inf Syst Res 19:253-272

Kannan K, Yun SH, Rudd RJ, Behr M (2010) High concentrations of persistent organic pollutants including PCBs, DDT, PBDEs and PFOS in little brown bats with white-nose syndrome in New York, USA. Chemosphere 80:613-618

Kaplan BS, O'Riain MJ, van Eeden R, King AJ (2011) A low-cost manipulation of food resources reduces spatial overlap between baboons (Papio ursinus) and humans in conflict. Int J Primatol 32:1397-1412

Kappeler PM, Van Schaik CP (2002) Evolution of primate social systems. Int J Primatol 23:707-740

Kappeler PM, Cremer S, Nunn CL (2015) Sociality and health: impacts of sociality on disease susceptibility and transmission in animal and human societies. Philos Trans R Soc Lond Ser B Biol Sci 370:20140116

Kasper C, Voelk1 B (2009) A social network analysis of primate groups. Primates 50:343-256

Kaur T, Singh J (2009) Primate-parasitic zoonoses and anthropozoonoses: a literature review. In: Huffman MA, Chapman CA (eds) Primate parasite ecology: the dynamics and study of hostparasite relationships. Cambridge University Press, Cambridge, pp 199-230

Kaur T, Singh J, Humphrey C, Tong S, Clevenger D, Tan W, Szekely B, Wang Y, Li Y, Alex Muse E, Kiyono M, Hanamura S, Inoue E, Nakamura M, Huffman MA, Jiang B, Nishida T (2008) Descriptive epidemiology of fatal respiratory outbreaks and detection of a human-related metapneumovirus in wild chimpanzees (Pan troglodytes) at Mahale Mountains National Park, western Tanzania. Am J Primatol 70:755-765

Kaur T, Singh J, Huffman MA, Petrzelkova KJ, Taylor NS, Xu S, Dewhirst FE, Paster BJ, Debruyne L, Vandamme P, Fox JG (2011) Campylobacter troglodytes sp. nov., isolated from feces of human-habituated wild chimpanzees (Pan troglodytes schweinfurthii) in Tanzania. Appl Environ Microbiol 77:2366-2373

Keeling MJ (1999) The effects of local spatial structure on epidemiological invasions. Proc Biol Sci 266:859-867

Keeling MJ (2005) The implications of network structure for epidemic dynamics. Theor Popul Biol 67:1-8

Kilonzo C, Atwill ER, Mandrell R, Garrick M, Villanueva V (2011) Prevalence and molecular characterization of Escherichia coli $\mathrm{O} 157: \mathrm{H} 7$ by multiple locus variable number tandem repeat analysis and pulsed field gel electrophoresis in three sheep farming operations in California. $\mathrm{J}$ Food Prot 74:1413-1421

Klovdahl AS (1985) Social networks and the spread of infectious diseases: the AIDS example. Soc Sci Med 21:1203-1216

Kohler TA, Gumerman GJ (2000) Dynamics of human and primate societies: agent-based modeling of social and spatial processes. Oxford University Press, Oxford 
Kowalewski MM, Salzer JS, Deutsch JC, Raño M, Kuhlenschmidt MS, Gillespie TR (2011) Black and gold howler monkeys (Alouatta caraya) as sentinels of ecosystem health: patterns of zoonotic protozoa infection relative to degree of human-primate contact. Am J Primatol 73:75-83

Krause J, Croft DP, James R (2007) Social network theory in the behavioural sciences: potential applications. Behav Ecol Sociobiol 62:15-27

Kumara HN, Singh M, Sharma AK, Santhosh K, Pal A (2014) Impact of forest fragment size on between-group encounters in lion-tailed macaques. Primates 55:543-548

Kyes R, Iskandar E, Onibala J, Paputungan U, Laatung S, Huettmann F (2012) Long-term population survey of the Sulawesi black macaques (Macaca nigra) at Tangkoko Nature Reserve, North Sulawesi, Indonesia. Am J Primatol 75:88-94

Laliberte E, Tylianakis JM (2010) Deforestation homogenizes tropical parasitoid-host networks. Ecology 91:1740-1747

Lane KE, Holley C, Hollocher H, Fuentes A (2011) The anthropogenic environment lessens the intensity and prevalence of gastrointestinal parasites in Balinese long-tailed macaques (Macaca fascicularis). Primates 52:117-128

Latapy M, Magnien C, Del Vecchio N (2008) Basic notions for the analysis of large two-mode networks. Soc Networks 30:31-48

Lee K, Divis PCS, Zakaria SK, Matusop A, Julin RA, Conway DJ, Cox-Singh J, Singh B (2011) Plasmodium knowlesi: reservoir hosts and tracking the emergence in humans and macaques. PLoS Pathog 7:1-11

Leendertz SAJ, Wich SA, Ancrenaz M, Bergl RA, Gonder MK, Humle T, Leendertz FH (2017) Ebola in great apes-current knowledge, possibilities for vaccination, and implications for conservation and human health. Mammal Rev 47:98-111

Li J (1999) The Tibetan macaque society: a field study. Anhui University Press, Hefei

Liu J, Dietz T, Carpenter SR, Alberti M, Folke C, Moran E et al (2007) Complexity of coupled human and natural systems. Science 317(5844):1513-1516

Loudon JE, Patel ER, Faulkner C, Schopler R, Kramer RA, Williams CV, Herrera JP (2017) An ethnoprimatological assessment of human impact on the parasite ecology of silky sifaka (Propithecus candidus). In: Fuentes A, Riley EP, Dore KM (eds) Ethnoprimatology: a practical guide to research at the human-nonhuman primate interface. Cambridge University Press, Cambridge, pp 89-110

Lowe SJ, Browne M, Boudjelas S (2000) Published by the IUCN/SSC Invasive Species Specialist Group (ISSG), Auckland

Lusseau D, Newman MEJ (2004) Identifying the role that individual animals play in their social network. Proc Biol Sci 271:S477-S481

MacIntosh AJJ (2016) Pathogen. In: Fuentes A, Bezanson M, Campbell CJ (eds) The international encyclopedia of primatology. Wiley, Hoboken, NJ

MacIntosh AJJ, Frias L (2016) Coevolution of hosts and parasites. In: Fuentes A, Bezanson M, Campbell CJ (eds) The international encyclopedia of primatology. Wiley, Hoboken, NJ

MacIntosh AJJ, Hernandez AD, Huffman MA (2010) Host age, sex and reproduction affect nematode parasitism among wild Japanese macaques. Primates 51:353-364

MacIntosh AJJ, Jacobs A, Garcia C, Shimizu K, Mouri K, Huffman MA, Hernandez AD (2012) Monkeys in the middle: parasite transmission through the social network of a wild primate. PLoS One 7:e51144

Marechal L, Semple S, Majolo B, Qarro M, Heistermann M, MacLarnon A (2011) Impacts of tourism on anxiety and physiological stress levels in wild male Barbary macaques. Biol Conserv 144:2188-2193

Marechal L, Semple S, Majolo B, MacLarnon A (2016) Assessing the effects of tourist provisioning on the health of wild Barbary macaques in Morocco. PLoS One 11:e0155920

Marty PR, Beisner B, Kaburu SSK, Balasubramaniam KN, Bliss-Moreau E, Ruppert N, Sah S, Ahmad I, Arlet ME, Atwill RA, McCowan B (2019) Time constraints imposed by anthropogenic environments alter social behaviour in long-tailed macaques. Anim Behav 150:157-165 
Matheson MD, Sheeran LK, Li J, Wagner S (2013) Tourist impact on Tibetan macaques. Anthrozoos 26:158-168

McCabe CM, Nunn CL (2018) Effective network size predicted from simulations of pathogen outbreaks through social networks provides a novel measure of structure-standardized group size. Front Vet Sci 5:71

McLennan MR, Mori H, Mahittikorn A, Prasertbun R, Hagiwara K, Huffman MA (2017) Zoonotic enterobacterial pathogens detected in wild chimpanzees. EcoHealth 15:143-147

Meyers LA (2007) Contact network epidemiology: bond percolation applied to infectious disease prediction and control. Bull Am Math Soc 44:63-86

Minsky M (1965) Matter, mind, and models. In: Minsky M (ed) Semantic information processing. MIT Press, Cambridge, pp 425-432

Moore C, Newman MEJ (2000) Epidemics and percolation in small-world networks. Phys Rev E 61:5678-5682

Moyes CL, Henry AJ, Golding N, Huang Z, Singh B, Baird JK, Newton PA, Huffman MA, Duda KA, Drakeley CJ, Elyazar IRF, Anstey NM, Chen Q, Zommers Z, Bhatt S, Gething PW, Hay SI (2014) Defining the geographical range of the Plasmodium knowlesi reservoir. PLoS Negl Trop Dis 8:e2780

Muehlenbein MP, Ancrenaz M (2010) Minimizing pathogen transmission at primate ecotourism destinations: the need for input from travel medicine. J Travel Med 16:229-232

Nahallage CAD, Huffman MA (2013) Macaque-human interactions in past and present-day Sri Lanka. In: Radhakrishna S, Huffman MA, Sinha A (eds) The macaque connection: cooperation and conflict between humans and macaques. Springer, New York, pp 135-148

Nahallage CAD, Huffman MA, Kuruppu N, Weerasingha T (2008) Diurnal primates of Sri Lanka and people's perception of them. Primate Conserv 23:1-7

Nesse RM, Bergstrom CT, Ellison PT, Flier JS, Gluckman P, Govindaraju DR, Niethammer D, Omenn GS, Perlman RL, Schwartz MD, Thomas MG, Stearns SC, Valle D (2010) Making evolutionary biology a basic science for medicine. PNAS 107:1800-1807

Newman MEJ (2002) Spread of epidemic disease on networks. Phys Rev E66:016128

Newman MEJ (2004) Analysis of weighted networks. Phys Rev E 70:056131

Newman MEJ (2006) Finding community structure in networks using the eigenvectors of matrices. Phys Rev E 74:036104

Nunn CL (2009) Using agent-based models to investigate primate disease ecology. In: Huffman M, Chapman CA (eds) Primate parasite ecology: the dynamics and study of host-parasite relationships. Cambridge University Press, Cambridge, pp 83-110

Nunn CL (2011) The comparative approach in evolutionary anthropology and biology. University of Chicago Press, Chicago

Nunn CL (2012) Primate disease ecology in comparative and theoretical perspective. Am J Primatol 74:497-509

Nunn CL, Altizer SM (2005) The global mammal parasite database: an online resource for infectious disease records in wild primates. Evol Anthropol 14:1-2

Nunn CL, Altizer SM (2006) Infectious diseases in primates: behavior, ecology and evolution. Oxford University Press, Oxford

Nunn CL, Thrall PH, Stewart K, Harcourt AH (2008) Emerging infectious diseases and animal social systems. Evol Ecol 22:519-543

Nunn CL, Thrall PH, Leendertz FH, Boesch C (2011) The spread of fecally transmitted parasites in socially structured populations. PLoS One 6:e21677

Nunn CL, Jordan F, McCabe CM, Verdolin JL, Fewell JH (2015) Infectious disease and group size: more than just a numbers game. Philos Trans R Soc Lond Ser B Biol Sci 370:20140111

Palacios JFG, Engelhardt A, Agil M, Hodges K, Bogia R, Waltert M (2012) Status of, and conservation recommendations for, the critically endangered crested black macaque Macaca nigra in Tangkoko, Indonesia. Oryx 46:290-297

Pasquaretta C, Levé M, Claidière N, van de Waal E, Whiten A, MacIntosh AJJ, Pele M, Bergstrom ML, Borgeaud C, Brosnan S, Crofoot MC, Fedigan LM, Fichtel C, Hopper LM, Mareno MC, 
Petit O, Schnoell AV, di Sorrentino EP, Thierry B, Tiddi B, Sueur C (2014) Social networks in primates: smart and tolerant species have more efficient networks. Sci Rep 4:7600

Paterson JD, Wallis J (2005) Commensalism and conflict: the human-primate interface. American Society of Primatologists, Norman, OK

Patterson JEH, Ruckstuhl KE (2013) Parasite infection and host group size: a meta-analytical review. Parasitology 140:803-813

Petrášová J, Petrželková KJ, Huffman MA, Mapua MI, Bobáková L, Mazoch V, Singh J, Kaur T, Petrášová KJ (2010) Gastrointestinal parasites of indigenous and introduced primate species of Rubondo Island National Park, Tanzania. Int J Primatol 31:920-936

Petrášová J, Uzliková M, Kostka M, Petrželková KJ, Huffman MA, Modrý D (2011) Diversity and host specificity of Blastocystis in syntopic primates on Rubondo Island, Tanzania. Int J Primatol 11:1113-1120

Phillips KA, Bales KL, Capitanio JP, Conley A, Czoty PW, Hart BA, Hopkins WD, Hu SL, Miller LA, Nader MA, Nathanielsz PW, Rogers J, Shively CA, Voytko ML (2014) Why primate models matter. Am J Primatol 76:801-827

Poirotte C, Massol F, Herbert A, Willaume E, Bomo PM, Kappeler PM, Charpentier MJE (2017) Mandrills use olfaction to socially avoid parasitized conspecifics. Sci Adv 3:e1601721

Poirotte C, Sarabian C, Ngoubangoye B, MacIntosh AJJ, Charpentier M (2019) Faecal avoidance differs between the sexes but not with nematode infection risk in mandrills. Anim Behav 149:97-106

Postel SL, Daily GC, Ehrlich PR (1996) Human appropriation of renewable fresh water. Science 271:785-788

Poulin R, Krasnov BR, Pilosof S, Thieltges DW (2013) Phylogeny determines the role of helminth parasites in intertidal food webs. J Anim Ecol 82:1265-1275

Priston NEC, McLennan MR (2013) Managing humans, managing macaques: human-macaque conflict in Asia and Africa. In: Radhakrishna S, Huffman MA, Sinha A (eds) The macaque connection, developments in primatology: progress and prospects. Springer, New York, pp $225-250$

Priston NEC, Wyper RM, Lee PC (2012) Buton macaques (Macaca ochreata brunnescens): crops, conflict, and behavior on farms. Am J Primatol 74:29-36

Radhakrishna S, Sinha A (2011) Less than wild? Commensal primates and wildlife conservation. J Biosci 36:749-753

Radhakrishna S, Huffman MA, Sinha A (2013) The macaque connection. Springer, New York

Ram S, Venkatachalam S, Sinha A (2003) Changing social strategies of wild female bonnet macaques during natural foraging and on provisioning. Curr Sci 84:780-790

Rezende EL, Albert EM, Fortuna MA, Bascompte J (2009) Compartments in a marine food web associated with phylogeny, body mass, and habitat structure. Ecol Lett 12:779-788

Rifkin J, Nunn CL, Garamszegi LZ (2012) Do animals living in larger groups experience greater parasitism? A meta-analysis. Am Nat 180:70-82

Riley EP (2003) "Whose woods are these?" Ethnoprimatology and conservation in Sulawesi, Indonesia. Am J Phys Anthropol:179-179

Riley EP (2007) The human-macaque interface: conservation implications of current and future overlap and conflict in lore Lindu National Park, Sulawesi, Indonesia. Am Anthropol 109:473-484

Riley EP, Fuentes A (2011) Conserving social-ecological systems in indonesia: human-nonhuman primate interconnections in Bali and Sulawesi. Am J Primatol 73:62-74

Rimbach R, Bisanzio D, Galvis N, Link A, Di Fiore A, Gillespie TR (2015) Brown spider monkeys (Ateles hybridus): a model for differentiating the role of social networks and physical contact on parasite transmission dynamics. Philos Trans R Soc Lond Ser B Biol Sci 370:20140110

Romano V, Duboscq J, Sarabian C, Thomas E, Sueur C, MacIntosh AJJ (2016) Modeling infection transmission in primate networks to predict centrality-based risk. Am J Primatol 78:767-779

Romano V, Shen M, Pansanel J, MacIntosh AJJ, Sueur C (2018) Social transmission in networks: global efficiency peaks with intermediate levels of modularity. Behav Ecol Sociobiol 72:154 
Rushmore J, Caillaud D, Hall RJ, Stumpf RM, Meyers LA, Altizer S (2014) Network based vaccination improves prospects for disease control in wild chimpanzees. J R Soc Interface 11:0349

Rushmore J, Bisanzio D, Gillespie TR (2017) Making new connections: insights from primateparasite networks. Trends Parasitol 33:547-560

Rwego IB, Isabirye-basuta G, Gillespie TR, Goldberg T (2007) Gastrointestinal bacterial transmission among humans, mountain gorillas, and livestock in Bwindi Impenetrable National Park, Uganda. Conserv Biol 22:1600-1607

Rwego IB, Gillespie TR, Isabirye-basuta G, Goldberg TL (2008) High rates of Escherichia coli transmission between livestock and humans in rural Uganda. J Clin Microbiol 46:3187-3191

Salathe M, Jones JH (2010) Dynamics and control of diseases in networks with community structure. PLoS Comput Biol 6:e1000736

Sapolsky RM, Romero LM, Munck AU (2000) How do glucocorticoids influence stress responses? Integrating permissive, suppressive, stimulatory, and preparative actions. Endocr Rev 21:55-89

Sarabian C, MacIntosh AJJ (2015) Hygienic tendencies correlate with low geohelminth infection in free-ranging macaques. Biol Lett 11:20150757

Sarabian C, Ngoubangoye B, MacIntosh AJJ (2017) Avoidance of biological contaminants through sight, smell and touch in chimpanzees. R Soc Open Sci 4:170968

Schwabe C (1984) Medicine and human health. William \& Wilkins, Baltimore

Sears HJ, Brownlee I, Uchiyama JK (1950) Persistence of individual strains of Escherichia coli in the intestinal tract of man. J Bacteriol 59:293-301

Sears HJ, Janes H, Saloum R, Brownlee I, Lamoreaux LF (1956) Persistence of individual strains of Escherichia coli in man and dog under varying conditions. J Bacteriol 71:370-372

Sha JCM, Gumert MD, Lee B, Jones-Engel L, Chan S, Fuentes A (2009) Macaque-human interactions and the societal perceptions of macaques in Singapore. Am J Primatol 71:825-839

Shah KV, Southwick CH (1965) Prevalence of antibodies to certain viruses in sera of free-living rhesus and of captive monkeys. Indian J Med Res 53:488-500

Siex KS (2005) Habitat destruction, population compression, and overbrowsing by the Zanzibar red colobus monkey (Procolobus kirkii). In: Paterson JD, Wallis J (eds) Commensalism and conflict: the human-primate interface. American Society of Primatologists, Norman, OK, pp 294-337

Silk MJ, Croft DP, Delahay RJ, Hodgson DJ, Boots M, Weber N, McDonald RA (2017) Using social network measures in wildlife disease ecology, epidemiology, and management. Bioscience 67:245-257

Singh M, Kumara HN, Kumar MA, Sharma AK (2001) Behavioural responses of lion-tailed macaques (Macaca silenus) to a changing habitat in a tropical rain forest fragment in the Western Ghats, India. Folia Primatol 72:278-291

Singh B, Kim Sung L, Matusop A, Radhakrishnan A, Shamsul SS, Cox-Singh J, Thomas A, Conway DJ (2004) A large focus of naturally acquired Plasmodium knowlesi infections in human beings. Lancet 363:1017-1024

Sinha A, Mukhopadhyay K, Datta-Roy A, Ram S (2005) Ecology proposes, behaviour disposes: ecological variability in social organization and male behavioural strategies among wild bonnet macaques. Curr Sci 89:1166-1179

Sinton L, Hall C, Braithwaite R (2007) Sunlight inactivation of Campylobacter jejuni and Salmonella enterica, compared with Escherichia coli, in seawater and river water. J Water Health 5:357-365

Southwick CH, Siddiqi MF (1994) Primate commensalism: the rhesus monkey in India. Rev Ecol 49:223-231

Southwick CH, Siddiqi MF (1998) The rhesus monkey's fall from grace. In: Ciochon RL, Nisbett RA (eds) The primate anthology. Prentice Hall, Upper Saddle River, pp 211-218

Southwick CH, Siddiqi F (2011) India's rhesus population: protection versus conservation management. In: Gumert MD, Fuentes A, Jones-Engel L (eds) Monkeys on the edge: ecology and 
management of long-tailed macaques and their interface with humans. Cambridge University Press, Cambridge, pp 275-292

Southwick CH, Siddiqi MF, Oppenheimer JR (1983) Twenty-year changes in rhesus macaque populations in agricultural areas of Northern India. Ecology 64:434-439

Spiesman BJ, Gratton C (2016) Flexible foraging shapes the topology of plant-pollinator interaction networks. Ecology 97:1431-1441

Springer A, Mellmann A, Fichtel C, Kappeler PM (2016) Social structure and Escherichia coli sharing in a group-living wild primate, Verreaux's sifaka. BMC Ecol 16:6

Stang M, Klinkhamer PGL, Waser NM, Stang I, van der Meijden E (2009) Size-specific interaction patterns and size matching in a plant-pollinator interaction web. Ann Bot 103:1459-1469

Stephens PR, Pappalardo P, Huang S, Byers JE, Farrell MJ, Gehman A, Ghai RR, Haas SE, Han B, Park AW, Schmidt JP, Altizer S, Ezenewa VO, Nunn CL (2017) Global mammal parasite database version 2.0. Ecology 98(5):1476

Sterck EHM (1998) Female dispersal, social organization, and infanticide in langurs: are they linked to human disturbance? Am J Primatol 44(4):235-254

Sueur C, Petit O, De Marco A, Jacobs AT, Watanabe K, Thierry B (2011a) A comparative network analysis of social style in macaques. Anim Behav 82(4):845-852

Sueur C, Jacobs A, Amblard F, Petit O, King AJ (2011b) How can social network analysis improve the study of primate behavior? Am J Primatol 73:703-719

Sueur C, Deneubourg JL, Petit O (2012) From social network (centralized vs. decentralized) to collective decision-making (unshared vs. shared consensus). PLoS One 7:e32566

Sueur C, Romano V, Sosa S, Puga-Gonzalez I (2018) Mechanisms of network evolution: a focus on socioecological factors, intermediary mechanisms, and selection pressures. Primates 60:1-15

Suleyman G, Tibbetts R, Perri MB, Vager D, Xin Y, Reyes K, Samuel L, Chami E, Starr P, Pietsch J, Zervos MJ, Alangaden G (2016) Nosocomial outbreak of a novel extended-spectrum beta-lactamase Salmonella enterica serotype isangi among surgical patients. Infect Control Hosp Epidemiol 37:954-961

Tenaillon O, Skurnik D, Picard B, Denamur E (2010) The population genetics of commensal Escherichia coli. Nat Rev Microbiol 8:207-217

Thierry B (2007a) The macaques: a double-layered social organization. In: Campbell CJ, Fuentes A, MacKinnon KC, Panger M, Bearder SK, Stumpf RM (eds) Primates in perspective. Oxford University Press, New York, pp 224-239

Thierry B (2007b) Unity in diversity: lessons from macaque societies. Evol Anthropol 16:224-238

Thierry B (2013) The macaques: a double-layered social organization. In: Campbell CJ, Fuentes A, MacKinnon KC, Bearder SK, Stumpf RM (eds) Primates in perspective. Oxford University Press, Oxford, pp 229-240

Thierry B, Aureli F, Nunn CL, Petit O, Abegg C, de Waal FBM (2008) A comparative study of conflict resolution in macaques: insights into the nature of covariation. Anim Behav 75:847-860

Tutin CEG (2000) Ecologie et organisation des primates de la foret tropicale africaine: aide a la comprehension de la transmission des retrovirus. For Trop Emerg Virales 93:157-161

Vallo P, Petrželková KJ, Profousová I, Petrášov J, Pomajbíková K, Leendertz F, Hashimoto C, Simmons N, Babweteera F, Piel A, Robbins MM, Boesch C, Sanz C, Morgan D, Sommer V, Furuichi T, Fujita S, Matsuzawa T, Kaur T, Huffman MA, Modry D (2012) Molecular diversity of entodiniomorphid ciliate Troglodytella abrassarti and its coevolution with chimpanzees. Am J Phys Anthropol 48:525-533

VanderWaal KL, Ezenwa VO (2016) Heterogeneity in pathogen transmission: mechanisms and methodology. Funct Ecol 30:1606-1622

VanderWaal KL, Atwill ER, Isbell LA, McCowan B (2013) Linking social and pathogen transmission networks using microbial genetics in giraffe (Giraffa camelopardalis). J Anim Ecol 83:406-414

VanderWaal KL, Atwill ER, Isbell LA, McCowan B (2014) Quantifying microbe transmission networks for wild and domestic ungulates in Kenya. Biol Conserv 169:136-146 
Vazquez DP, Chacoff N, Cagnolo L (2009) Evaluating multiple determinants of the structure of plant-animal mutualistic networks. Ecology 90:2039-2046

Waters CN, Zalasiewicz J, Summerhayes C, Barnosky AD, Poirier C, Gauszka A, Cearreta A, Edgeworth M, Ellis EC, Ellis M, Jeandel C, Leinfelder R, McNeill JR, Richter DB, Steffen W, Syvitski J, Vidas D, Wagreich M, Williams M, Zhisheng A, Grinevald J, Odada E, Oreskes N, Wolfe AP (2016) The Anthropocene is functionally and stratigraphically distinct from the Holocene. Science 351:aad2622

Wenz-Mücke A, Sithithaworn P, Petney TN, Taraschewski H (2013) Human contact influences the foraging behaviour and parasite community in long-tailed macaques. Parasitology 140:709-718

Wey T, Blumstein DT, Shen W, Jordan F (2008) Social network analysis of animal behaviour: a promising tool for the study of sociality. Anim Behav 75:333-344

Whitehead H, Dufault S (1999) Techniques for analyzing vertebrate social structure using identified individuals: review and recommendations. Adv Study Behav 28:33-74

Wilensky U, Stroup W (1999) NetLogo HubNet Disease model [computer software]. Center for Connected Learning and Computer-Based Modeling, Northwestern University, Evanston, IL

Wolfe ND, Dunavan CP, Diamond J (2007) Origins of major human infectious diseases. Nature 447:279-283

Young C, Majolo B, Heistermann M, Schülke O, Ostner J (2014) Responses to social and environmental stress are attenuated by strong male bonds in wild macaques. PNAS 111:18195-18200

Zhang X, Kadir KA, Quintanilla-Zarinan LF, Villano J, Houghton P, Du H, Singh B, Glenn Smith D (2016) Distribution and prevalence of malaria parasites among long-tailed macaques (Macaca fascicularis) in regional populations across Southeast Asia. Malar J 15:450

Zhao QK (1996) Etho-ecology of Tibetan macaques at Mount Emei, China. In: Fa J, Lindburg DG (eds) Evolution and ecology of macaque societies. Cambridge University Press, Cambridge

Zhao QK (2005) Tibetan macaques, visitors, and local people at Mt. Emei: problems and countermeasures. In: Paterson JD, Wallis J (eds) Commensalism and conflict: the human-primate interface. American Society of Primatologists, Norman, OK, pp 376-399

Zinsstag J, Schelling E, Waltner-Toews D, Tanner M (2011) From "one medicine" to "one health" and systemic approaches to health and well-being. Prev Vet Med 101:148-156

Zinsstag J, Schelling E, Waltner-Toews D, Tanner M (2015) One health, the theory and practice of integrated one health approaches. CAB International, Oxfordshire

Open Access This chapter is licensed under the terms of the Creative Commons Attribution 4.0 International License (http://creativecommons.org/licenses/by/4.0/), which permits use, sharing, adaptation, distribution and reproduction in any medium or format, as long as you give appropriate credit to the original author(s) and the source, provide a link to the Creative Commons licence and indicate if changes were made.

The images or other third party material in this chapter are included in the chapter's Creative Commons licence, unless indicated otherwise in a credit line to the material. If material is not included in the chapter's Creative Commons licence and your intended use is not permitted by statutory regulation or exceeds the permitted use, you will need to obtain permission directly from the copyright holder.

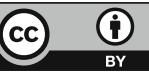

